\title{
NUMERICAL INVESTIGATION OF THE NONLINEAR DYNAMICS OF A HYBRID ACOUSTO-OPTIC BRAGG CELL WITH A VARIABLE FEEDBACK GAIN
}

Thesis

Submitted to

The School of Engineering of the UNIVERSITY OF DAYTON

In Partial Fulfillment of the Requirements for

The Degree of

Master of Science in Electrical Engineering

By

Hao Zhou

Dayton, Ohio

August 2014

\begin{tabular}{|} 
UNIVERSITY of \\
DA \\
DAYTON \\
\hline DOT
\end{tabular} 


\section{NUMERICAL INVESTIGATION OF THE NONLINEAR DYNAMICS OF A HYBRID ACOUSTO-OPTIC BRAGG CELL WITH A VARIABLE FEEDBACK GAIN}

Name: Zhou, Hao

APPROVED BY:

Monish R. Chatterjee, Ph.D

Advisory Committee Chairman

Professor

Electrical and Computer Engineering
Eric Balster, Ph.D

Committee Member

Professor

Electrical and Computer Engineering

Guru Subranmanyam, Ph.D

Committee Member

Department Chair and Professor

Electrical and Computer Engineering

John G. Weber, Ph.D

Associate Dean

School of Engineering
Eddy M.Rojas, Ph.D., M.A., P.E.

Dean, School of Engineering 
(C) Copyright by

Hao Zhou

All rights reserved

2014 


\begin{abstract}
NUMERICAL INVESTIGATION OF THE NONLINEAR DYNAMICS OF A HYBRID ACOUSTO-OPTIC BRAGG CELL WITH A VARIABLE FEEDBACK GAIN
\end{abstract}

Name: Zhou, Hao

University of Dayton

Advisor: Professor Monish. R. Chatterjee

Since around 1979, the operation of an acousto-optic Bragg cell under positive first-order feedback via amplification and delay in the loop has been studied extensively by several groups [1-3]. It is well-known that this system offers interesting dynamical properties, including bistability and chaos under a range of parameter thresholds. Thus, for fixed values of the bias voltage $\left(\hat{\alpha}_{0}\right)$, feedback gain $(\widetilde{\beta})$, feedback time delay (TD), incident optical intensity $\left(I_{\text {inc }}\right)$ and initial first-order intensity $\left(I_{l}(0)\right)$, the hybrid system may be operated in regimes where it is bistable (thus leading to possible logical applications), or chaotic (within which it may exhibit period-2, period-3 and similar characteristics). In recent work, the analysis of the system was extended to include bistable maps and bifurcation specific to chaotic regimes whereby chaotic passbands and stopbands were established. Additionally, the system was utilized for signal 
encryption and decryption applications under chaos for uniform plane waves. The present work originated with the problem of a variable aperture opening relative to the first-order light. This potentially complex problem is simplified by assuming instead a variable feedback gain $(\widetilde{\beta}(\mathrm{t}))$, which leads to considerably different nonlinear dynamics. This research explores initially the simpler stages of this problem by examining the nonlinear dynamics versus the (DC) bias voltage for different variable- $\widetilde{\beta}$ conditions, including very slow and likewise fast rates of change of the gain with time in relation to the feedback delay. It is found that the response depends critically on the rate of rise of the feedback gain, and also that the resulting chaotic regimes are generally significantly different from those for fixed values of $\widetilde{\beta}$. We have generated constant feedback gain $\quad \widetilde{\beta}$ and the variable feedback gain $\widetilde{\beta}(\mathrm{t})$ chaos characteristics of the hybrid A-O network. Chaos as an equivalent carrier has been used to encrypt messages, and this strategy may be used as a means of ensuring information security in keeping the information safe. A receiver scheme was developed using a second Bragg cell with a matched bias input to generate local chaos that was used to extract message using a heterodyne reception technique, followed by a low pass filter and a phase inverter. Results between variable- and fixed-gain systems are compared in terms of advantages and disadvantages. 


\section{ACKNOWLEDGEMENTS}

I would like to thank Dr. Monish Chatterjee, my research advisor, who gave me his time and resources and helped me considerably in my research. I appreciate his support in directing my research and help in making it beautiful.

I would also like to thank all the members who have helped me at various times during my research, my graduate class seniors who have helped me with my work.

At last, I also sincerely thank my parents. 


\section{TABLE OF CONTENTS}

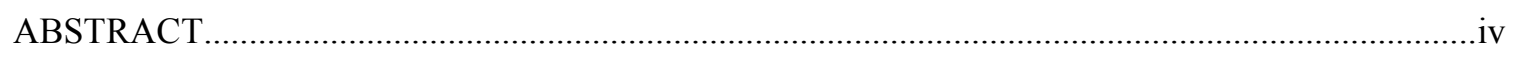

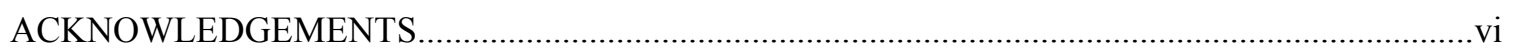

TABLE OF CONTENTS............................................................................................................. vii

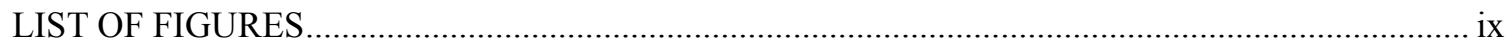

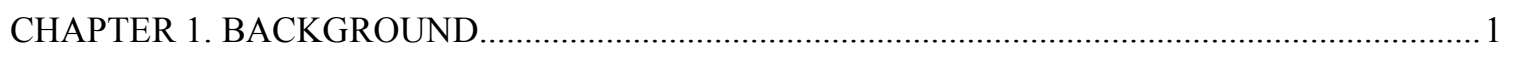

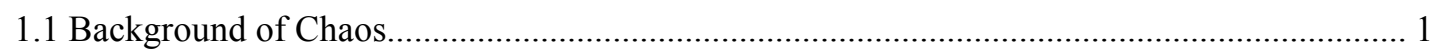

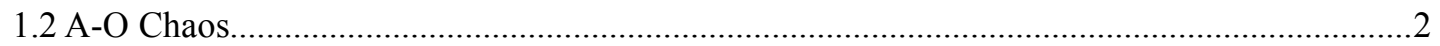

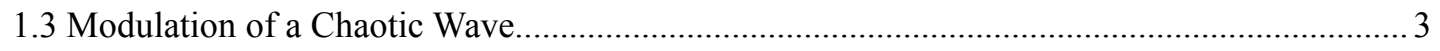

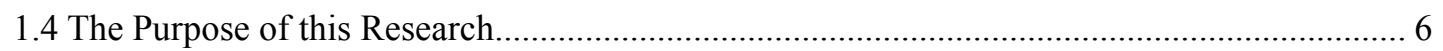

CHAPTER 2. ACOUSTO-OPTIC CHAOS : A SIMULATION STUDY OF GENERAL

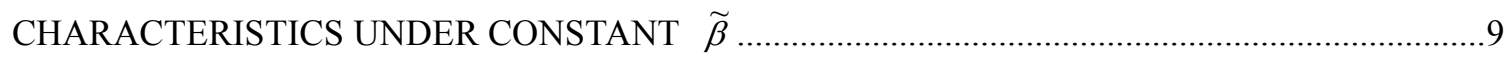

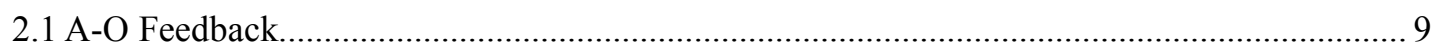

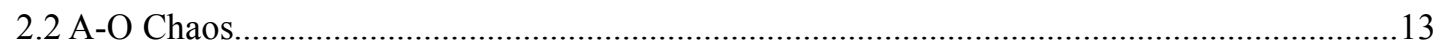

2.3 Examination of Dynamical Behavior Based on Bifurcation Maps.......................................... 19

CHAPTER 3. ACOUSTO-OPTIC CHAOS : A SIMULATION STUDY OF GENERAL

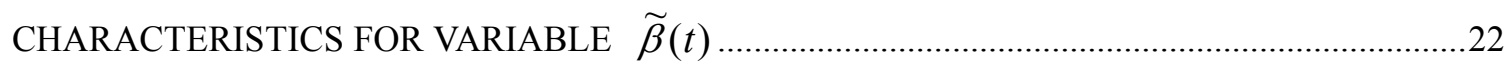

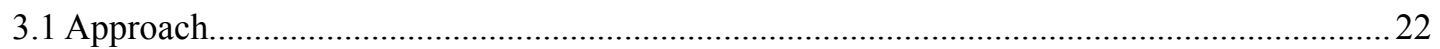


CHAPTER 4. CHAOTIC SIGNAL ENCRYPTION AND DECRYPTION AND THE

INFORMATION SECURITY OF THE VARIABLE FEEDBACK GAIN..................................... 28

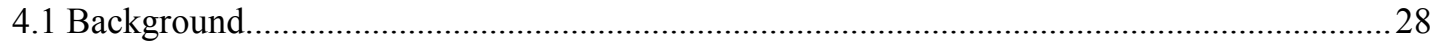

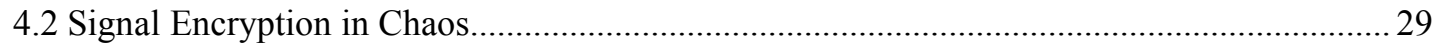

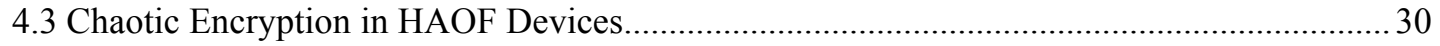

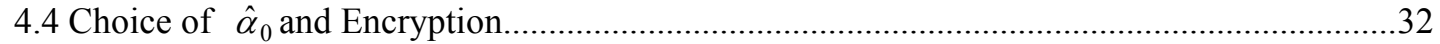

4.5 Receiver System and Decryption of Modulated Chaos................................................... 33

4.6 Chaotic Encryption and Decryption of the Constant Feedback Gain.................................. 34

4.7 Chaotic Encryption and Decryption of the Variable Feedback Gain................................... 36

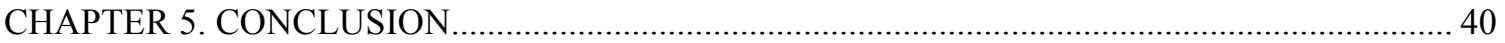

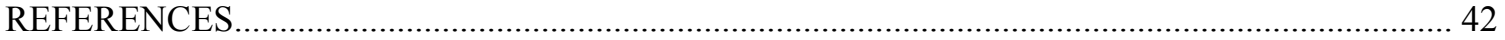




\section{LIST OF FIGURES}

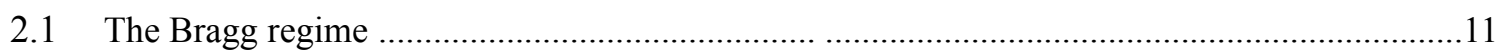

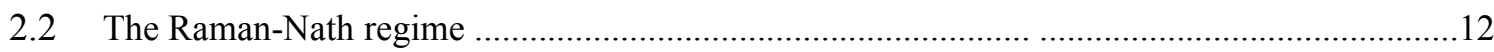

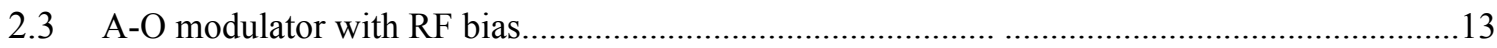

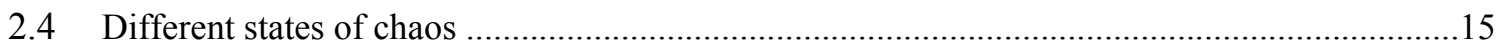

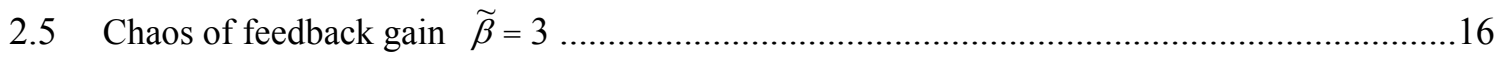

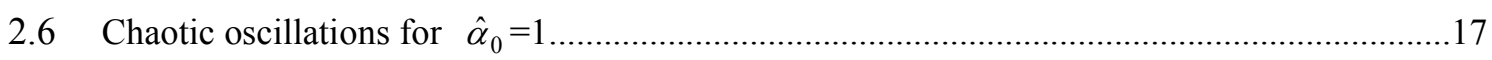

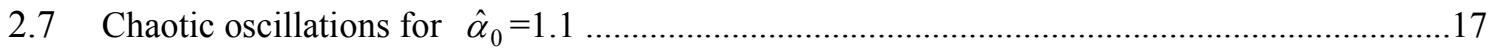

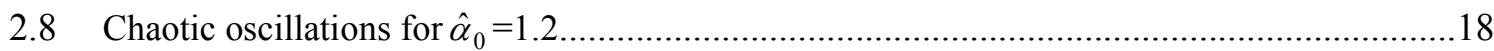

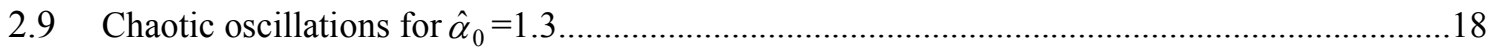

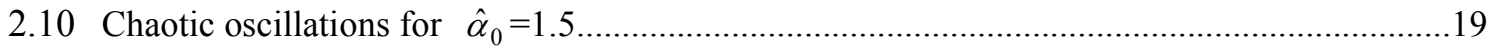

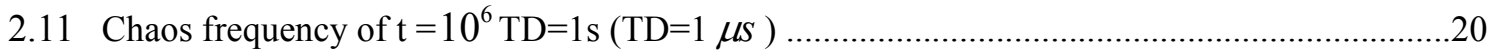

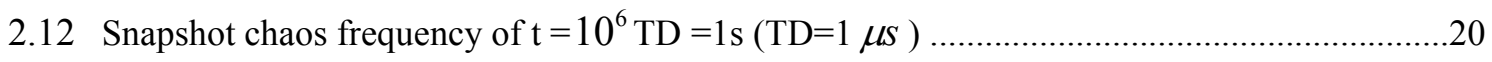

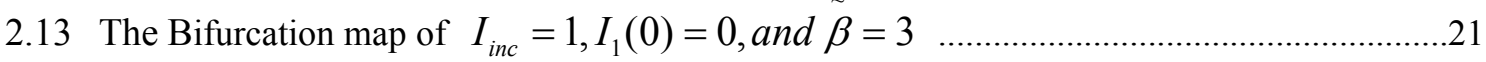

3.1 A-O Modulator with RF bias under variable feedback gain .......................................22

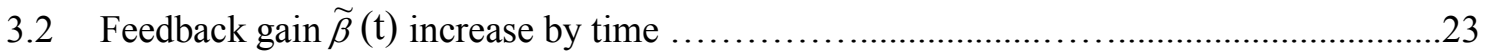

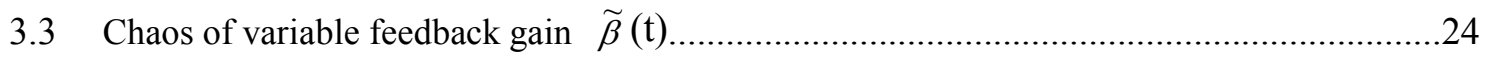


3.4 Variable feedback gain chaos frequency of $\mathrm{t}=10^{6} \mathrm{TD}=1 \mathrm{~s}(\mathrm{TD}=1 \mu s)$

3.5 Snapshot variable feedback gain chaos frequency of $\mathrm{t}=10^{6} \mathrm{TD}=1 \mathrm{~s}(\mathrm{TD}=1 \mu s) \ldots \ldots \ldots \ldots \ldots \ldots \ldots . . .25$

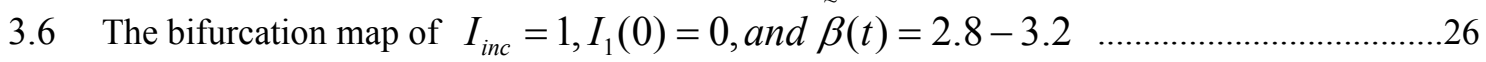

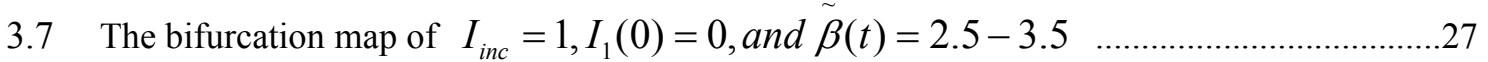

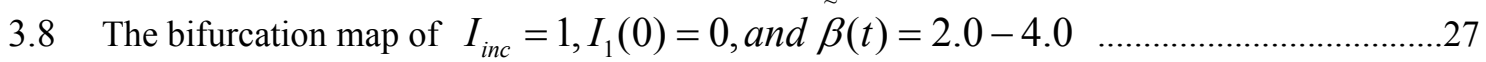

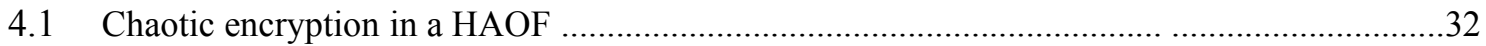

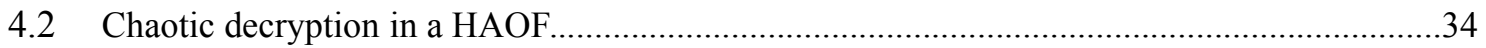

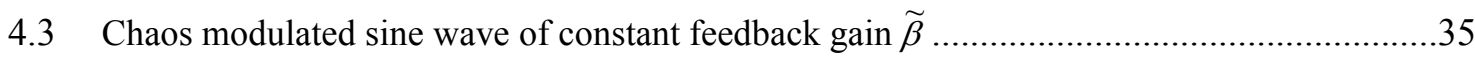

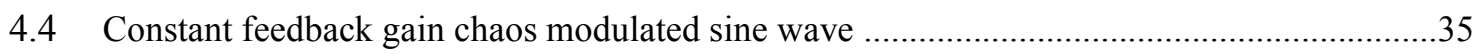

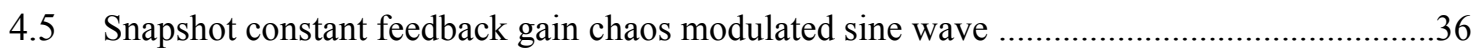

4.6 Comparison between filtered data and original signal for chaos modulation under

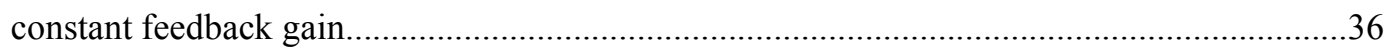

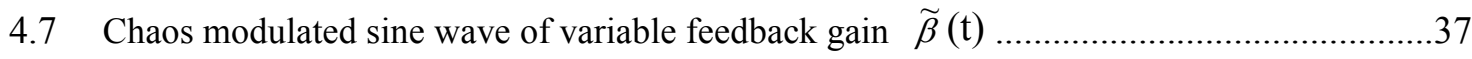

4.8 Variable feedback gain chaos modulated sine wave ..............................................................

4.9 Snapshot variable feedback gain chaos modulated sine wave ..............................................38

4.10 Comparison between filtered data and original signal for chaos under variable feedback gain .38 


\section{CHAPTER 1}

\section{BACKGROUND}

\subsection{Background of Chaos}

There is no precise definition of chaos except that chaos is a form of controlled oscillations, often appearing to be time-shifted resonances. Chaos has the form of random oscillations in time with non-uniformity in amplitude but essentially stable frequency characteristics. Study of chaos in the modern era dates back to late 1950s when experiments were conducted to study chaotic behavior using circuits, forced vibrations of shallow water waves in a finite container, hydro dynamical instabilities, chemical turbulence, and acousto-optic (A-O) turbulence [1].

Early experiments on chaos were conducted by Faraday using forced vibrations of shallow water waves in a finite container, the vibrations were driven into chaos when a component at a frequency $\mathrm{f}_{0} / 2$ in shallow water was observed when the medium was excited with a frequency of $f_{0}$. This was the likely demonstration of the first existence of chaos[2,3].

Conducting experiments using shallow water waves was expensive, used a lot of equipment and was difficult to analyze. The next set of experiments included the use of nonlinear circuits; this was obviously cheaper and could be built readily in a laboratory. 
These experiments used many coupled oscillators [4,5] but later researchers who used a single oscillator driven by a periodic signal[5-10].

In 1963, E.N Lorenz published several papers based on thermal model convection [11]. He observed that in a deterministic system of three ordinary differential equations the solutions were bounded but unstable and underwent irregular vibrations while being confined to the medium. The word "chaos" was first introduced to describe this phenomenon by Li and Yorke to denote the apparently random output of some mappings [12].

Though chaos was initially observed in nonlinear circuits, and shallow water experiments, it turns out that chaos can also be observed in nonlinear optical systems including laser beams, optical fibers and in acousto-optics. The importance of optical chaos in practical applications is inherent in the speed and parallelism of optical systems.

\subsection{A-O Chaos}

Acousto-optics, where acousto- implies sound and optics implies light defines the interaction of sound with light. Chaos can be generated and studied using a hybrid A-O chaos system. In 1979, Ikeda predicted that chaos can be observed in an optically bistable device using a ring cavity [13]. This predicted phenomenon was first successfully observed by Gibbs in 1981 [14]. A hybrid optical system implies that a part of the optical output is electronically delayed, amplified and fed back to the driver input. Periodic optical oscillations are generated in hybrid devices by introducing delay in the feedback line. This was established via the experiments conducted by Ikeda in ring cavity and delay 
line hybrid devices. All chaotic optical devices in general exhibit optical bistability [15]. Typically, optical bistability is defined through a set of coupled differential equations. The present research focuses on an A-O Bragg cell with a first-order feedback. The output of the Bragg cell consists of zeroth- and first-order light beams. The first-order light beam is detected, amplified and fed back to the input. The time delay in the feedback makes the system nonlinear and complex in space-time analysis [16-17]. The time delay also introduces bistability in the hybrid A-O feedback (HAOF) device. Monostability, bistability, possible multistability and chaos in the hybrid A-O device may be obtained via three tuning effects viz., the feedback gain, time delay and the bias input [18-19].

Generation of A-O chaos involves careful tuning of the parameters and amplification of the feedback signal. Another process of generating acoustic chaos involves sub-harmonic components in the spectra of cavities (bubble formation in a liquid subjected to high power supersonic irradiation) with intrinsic noise.

\subsection{Modulation of a Chaotic Wave}

A common motivation behind most communication systems is to transmit data in a safe and secure manner. Typically, a basic problem is information security. Chaos waves may be used as a carrier for transmitting data because of their random yet controlled nature. Modulation using chaos as a carrier wave was first attempted in the 1990s and many schemes have been designed to encrypt chaos waves using message signals with fewer numerical algorithms as encryption key than the classical methods [20,21]. The chaos wave may be viewed as a carrier which is 
subjected to a simple Amplitude Modulation (AM) scheme. Chaos may be viewed as a random disturbance that may be used to mask data from hackers. The basic random nature of chaos in the A-O case is inherent in its dependence on three factors viz. feedback gain, bias input and time delay that in effect protect the data. As discussed earlier, an A-O chaos system is governed by a key set of simple nonlinear differential equations that may be used to decrypt encrypted data transmitted over a channel. The resulting communication scheme using chaos as a carrier is similar in some respects to amplitude modulation, and therefore we may use a heterodyne scheme at the receiver for demodulation. The transmitter consists of a master chaos generator, a message generator and a device to encrypt the information on to the chaos carrier. The demodulation is conceptually similar to that of simple amplitude demodulation. The receiving scheme involves generating a chaos carrier with same characteristics as that of the input and using it via heterodyne to retrieve the information sent. Thus, the receiver system consists of a slave chaos generator (usually has the same characteristics as that of the transmitter) that generates the chaos carrier with the same frequency as that of the transmitted chaos carrier, a multiplier and a filtering device to filter out the unnecessary components in the output.

Pecora et.al [22] demonstrated the use of a stable and unstable subsystem to generate chaos. They used the same stable subsystem at the receiver to generate the chaos carrier synchronized to the original that may then be used for decoding the information sent. Chaotic modulation using chaos as a carrier wave provides a wide variety of defense applications. Another approach to modulating a chaos wave consists of encoding the information on unstable periodic orbits (UPOS) 
attached to the chaos in its phase space [23]. This encryption scheme uses each unstable periodic orbit to address separately the information signal. This is achieved by the so called OGY (Ott, Grebogi, Yorke [24]) method which addresses chaotic oscillations in any of its UPOs using the message signal. The decoding is carried out in a reciprocal manner in this method. Though the OGY method seems attractive, it takes a lot of processing time and equipment.

Several chaotic encryption schemes have been proposed in optoelectronic systems using the chaotic fluctuations of optical power generated by laser diodes for data encryption $[25,26]$. This type of chaos generation using laser diodes is highly complex and requires extensive high efficiency equipment. In general, this scheme can be used for encrypting data at a high bit rates over a wide bandwidth. This scheme generates high complexity chaos with fast intensity fluctuations. It is likely to find use in the future for highly reliable optical fiber communications. This scheme of generating chaos in power requires the physical parameters of the laser diodes such as nonlinear characteristics, and nonlinear behavior in power to be controlled accurately and with high reliability.

Larger et. al [20] measured chaos in terms of wavelength and then used this approach rather than using chaos as intensity to encrypt the signal waveforms. This scheme had high reliability and accuracy in transmitting as well as receiving the messages. At the receiver, the decoding was carried out by generating non-autonomous chaos in wavelength. Chaos in wavelength was achieved using a tunable laser diode, in which the wavelength agility depends on fine tuning the diode. The transmitter part contained a tunable laser diode, the laser beam, a photodetector, 
feedback with time delay, low pass filter and an adder circuit to add the feedback to the input message. The receiver had the same configuration except for the tunable laser diode. The differences between this approach and the current research are the use of chaos in intensity and in the feedback loop in the system. In this scheme the original message is recovered from the encrypted signal by multiplying the encrypted signal with a local chaos carrier and then low pass filtering the multiplier output to remove the carrier and retrieve the message.

This proposed research is based on fourth order Runge-kutta algorithm. The important parameters that model the whole system of this research are the feedback gain $\widetilde{\beta}$, input bias $\left(\hat{\alpha}_{0}\right)$ and the time delay (TD).

\subsection{The Purpose of this Research}

From the previous research carried out on this subject, many have successfully demonstrated the behavior of optical bistability and chaos [27] and how chaos behaves with time. Optical monostability, bistability and mutistability on the way to chaos have been successfully demonstrated in various papers by varying the parameters that affect the system. Since about 1998, research has demonstrated the use of optical chaos as a carrier for secure communication. Beginning in 2008, al-Saedi and Chatterjee [28] have applied this communication scheme to the hybrid A-O device using chaos in intensity. The research has generally established this communication system and proved its functionality. They have carried out the A-O chaos of a constant $\widetilde{\beta}$. The purpose in this research is to examine A-O chaos in the presence of a 
variable $\widetilde{\beta}$. The following is a partial list of tasks that were completed under this research.

1) Generating a chaos under constant $\widetilde{\beta}$;

2) Generating a chaos of variable $\widetilde{\beta}(t)$;

3) Successful encryption and decryption of basic analog waves such as a sinusoidal signal;

4) Transmission and reception of data using both constant and variable feedback gains.

The variable $\widetilde{\beta}$ problem originated with the concept of a variable aperture opening on the surface of the photodetector (PD) in the feedback system. Clearly, this would imply a time-varying difference in the photocurrent picked up by the PD, thereby potentially changing the basic nonlinear dynamics of the chaotic system. Since this problem is inherently rather complex, involving the use of Poynting vectors and the collected intensity over the aperture cross-section, it was decided that a more straightforward approach would be to instead consider a variable feedback gain, whereby the photocurrent would become time-varying in accordance with the rate of change of the gain. Initially, this problem posed certain difficulties, in particular relative to the rate of increase of the gain as compared with the feedback time delay. The resulting nonlinear dynamics (NLD) was essentially both intractable and non-usable. Later, after some iterations, it was found that the NLD becomes more well-defined under relatively slow rate of rise of the feedback gain, while revealing interesting differences from the fixed- $\widetilde{\beta}$ case. The limits of such behavior have been examined in some detail, and eventually optimal slopes and time durations have been established. Using data from these findings, a complete encryption and decryption analysis has been carried out using the variable- $\widetilde{\beta}$ simulation. The results are 
compared with the corresponding fixed- $\widetilde{\beta}$ problem (with identical system parameters), and it is found that the variable- $\widetilde{\beta}$ case (with proper control) may have potential advantages in terms of broader chaotic passbands (which are evident), and possibly better parameter tolerance. Chapter 2 provides a brief overview of chaos generation in HAOF under constant $\widetilde{\beta}$. The onset of chaotic oscillations and the corresponding NLD and time dynamics (the latter as part of this research) are examined in this chapter. 


\section{CHAPTER 2}

\section{ACOUSTO-OPTIC CHAOS : A SIMULATION STUDY OF GENERAL CHARACTERISTICS UNDER CONSTANT $\tilde{\beta}$}

\subsection{A-O Feedback}

A-O means interaction of sound with light. The phenomenon of acousto-optics is the diffraction of monochromatic light waves by ultrasonic sound and was first discovered by Brillouin in1921 [29]. Debye, Sears, Lucas and Biquard [30,31], have reported experiments illustrating the diffraction of light using high-frequency sound waves in a liquid. This arrangement was later described by a process involving monochromatic light passing through a rectangular structured cell and emerging from the other side [32]. When the medium is homogeneous and isotropic there is no deviation in the monochromatic light through the medium.

But when the medium is traversed with high frequency sound wave, the light wave is diffracted.

When a sound wave passes through the medium, it causes compressions and rarefactions in the medium manifested through the photo-elastic effect in the medium. When the monochromatic light is incident on the medium at an angle called the Bragg angle $\left(\theta_{B}\right)$, the light is diffracted into two orders, the first- and zeroth-orders [33]. The layers formed by the compressions and rarefactions in the medium travel at the velocity of sound incident on the medium. 
An acoustic wave is generated by applying an RF signal to a piezoelectric transducer that is suitably bonded to a crystal. The sound wave acts like a phase grating. An equivalent Figure of Merit Q similar to the quality factor in electrical resonance was introduced by Klein and Cook [34] that explains the diffraction in different A-O regimes.

$\mathrm{Q}$ is given by

$Q=\frac{L K^{2}}{k}=\frac{2 \pi \lambda_{0} L}{n \Lambda^{2}}=\frac{2 \pi \lambda_{0} L \Omega^{2}}{n v_{s}^{2}}$,

where,

Q - Klein-Cook Parameter

L - Effective legth of A-O Modulator

$\mathrm{n}$ - The index of refraction

$\lambda_{0}$ - Wavelength of light in free-space

$\Omega$ - Frequency of the sound wave

$v_{s}$ - Velocity of the sound wave

$\mathrm{k}$ - Wave number of light in medium

$\mathrm{K}$ - Wave number of sound

$\Lambda$ - Wavelength of sound inside the modulator

Q is an important factor which determines the regime of operation of an A-O modulator.

When $\mathrm{Q}$ is less than 1, the system operates in the Raman-Nath regime and when $\mathrm{Q}$ is greater than 1, the system operates in Bragg regime. In Bragg regime when the light is incident at an angle $\left(\theta_{B}\right)$ called the Bragg angle, the light undergoes diffraction and generates first- and zeroth-orders 
as mentioned above. In 1996, Chatterjee and Chen showed that Q must be greater than $8 \pi$ for the system to operate strictly in the Bragg regime [35]. The Bragg angle $\theta_{B}$ is approximately given by

$$
\theta_{B}=\frac{K}{2 k}
$$

The different regimes of operation are shown in Figs.2.1 and 2.2.

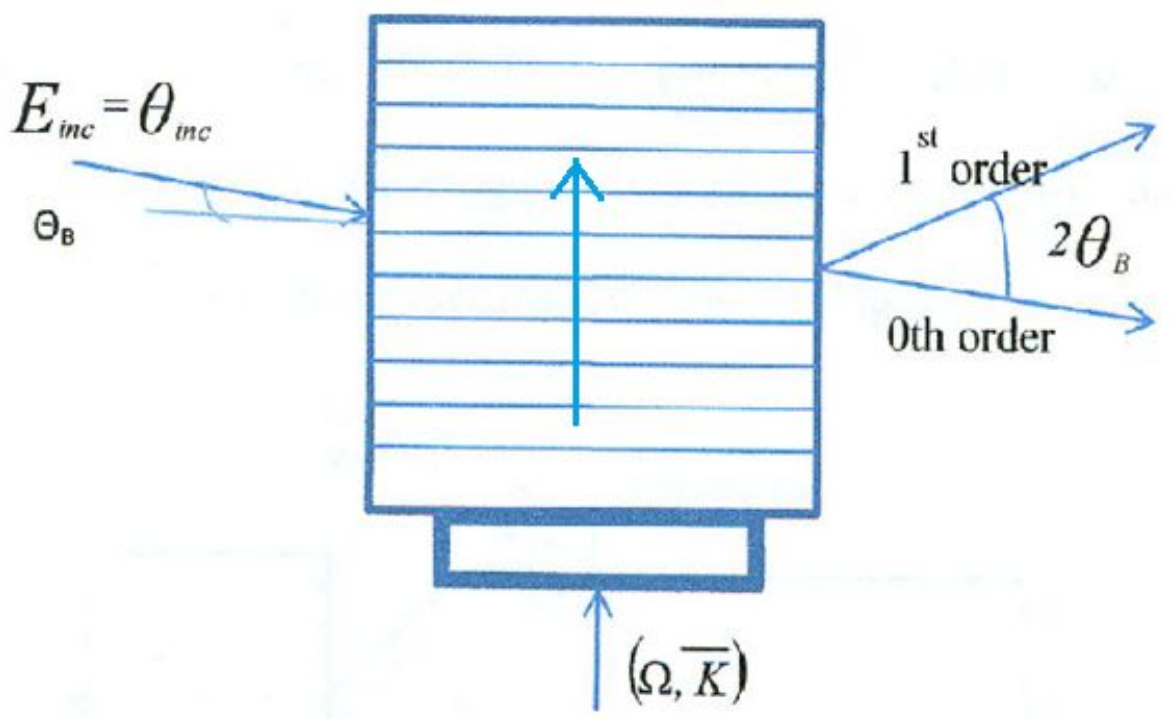

Fig.2.1. The Bragg regime. 


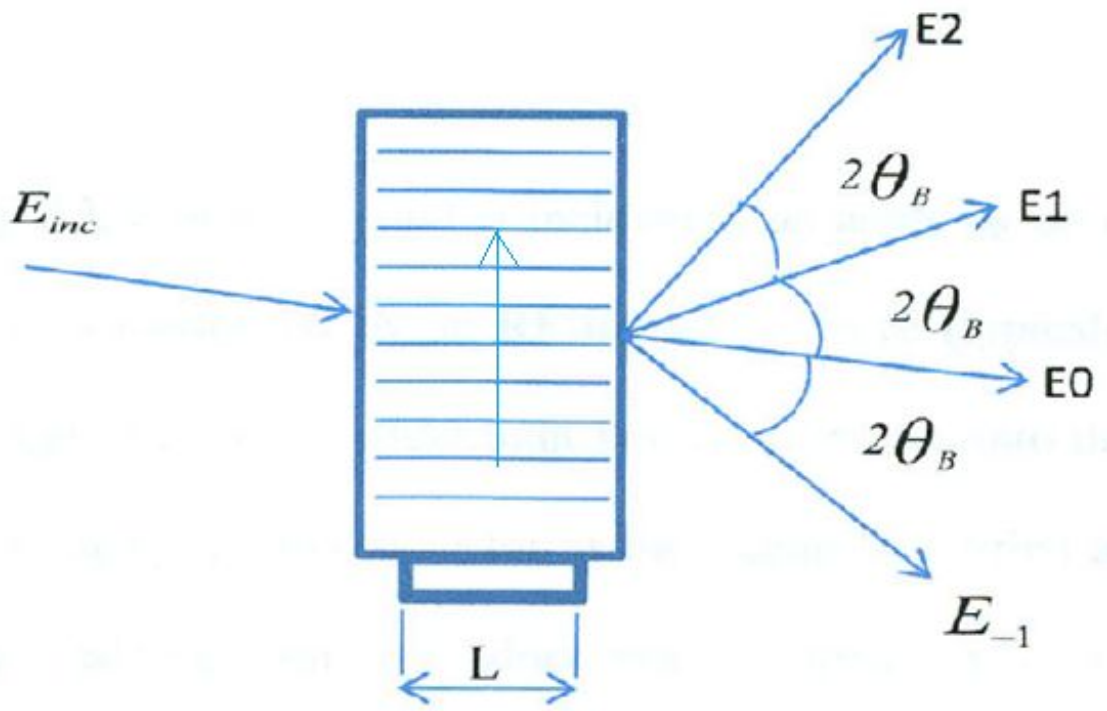

Fig.2.2 . The Raman-Nath regime.

Most experiments involving A-O feedback assume operation in the Bragg regime. Around 1978, it was discovered that an A-O network with positive feedback exhibits bistability and hysteresis characteristics [36]. In refs [17] and [18] Chrostowski et.al used equivalent circuit models of the Bragg regime to demonstrate A-O bistabiolity. The standard Bragg cell transmitter with feedback is shown in Fig.2.3. 


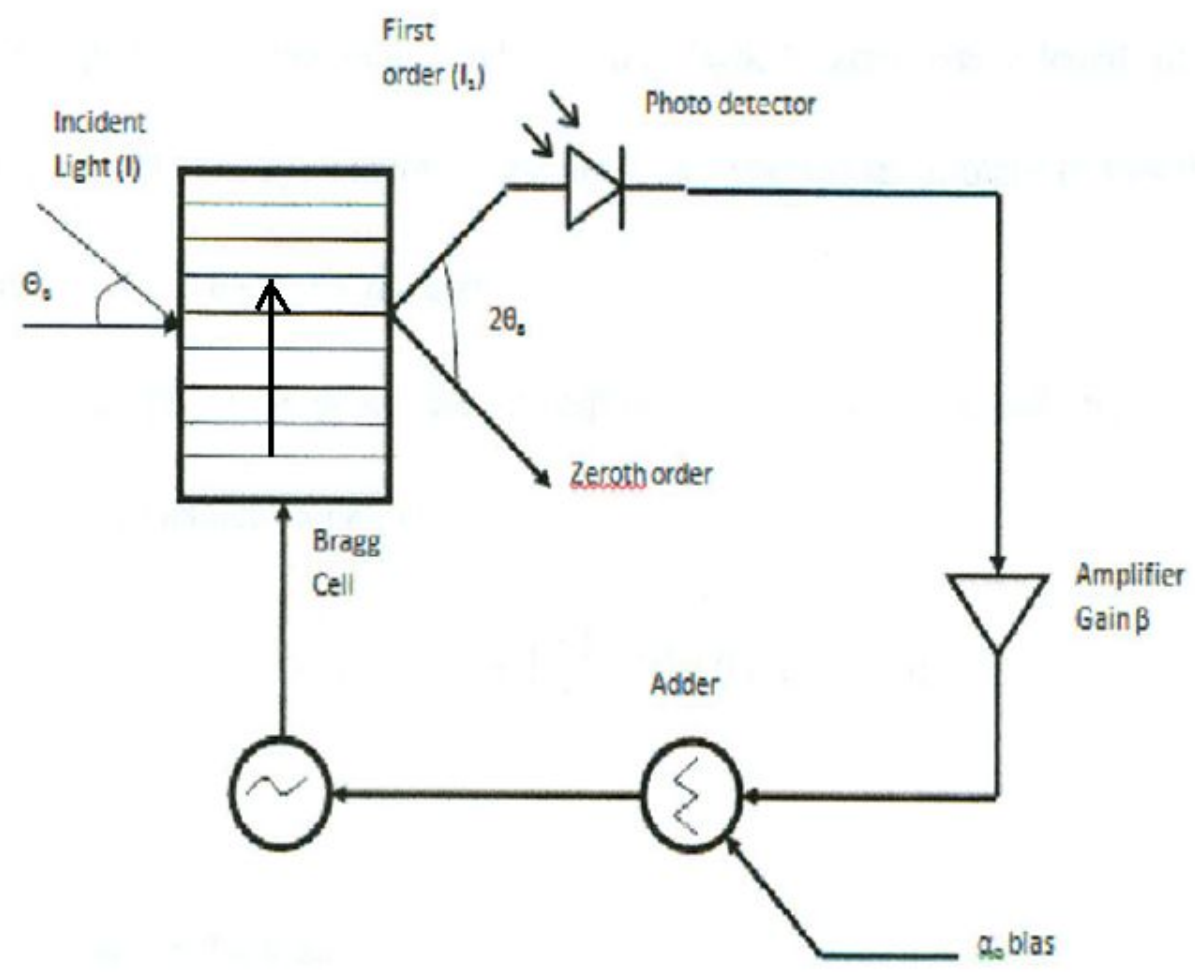

Fig.2.3. A-O Modulator with RF bias.

\subsection{A-O Chaos}

As shown in Fig.2.3, a beam of light is incident at an angle $\theta_{B}$ on a Bragg cell modulator, which in turn is modulated by an RF ultrasonic wave traveling through the Bragg cell. The incident light is diffracted into the first-order (intensity $I_{1}$ ) that is detected by a photo detector at the output, amplified and then fed back to the adder in the feedback loop. The adder combines the feedback with the dc bias input and feeds it to the RF source that generates the RF input which is then incident on the Bragg cell via a transducer. The plot of the first-order intensity vs. 
the bias input $\left(\hat{\alpha}_{0}\right)$ shows the monostability, bistability and chaotic conditions of the modulator. The operating region of an A-O modulator depends on three important factors: feedback gain $\widetilde{\beta}$, input bias $\left(\hat{\alpha}_{0}\right)$ and the time delay (TD).

With feedback plus time delay, the corresponding first-order detected intensity follows the nonlinear dynamical equation:

$$
I_{1}(t)=I_{i n c} \sin ^{2}\left[\frac{1}{2}\left(\hat{\alpha}_{0}(t)+\tilde{\beta} I_{1}(t-T D)\right)\right],
$$

where

$\widetilde{\beta}-\quad$ effective feedback gain;

$I_{i n c}$ - incident intensity;

TD - feedback time delay including photo detector conversion delay;

$\hat{\alpha}_{0}-$ bias input.

A study of the time characteristics of A-O chaos is necessary for the understanding of the oscillating behavior of chaos. An A-O system operates in monostable, bistable and chaotic regions depending on the bias input as was discussed earlier. Fig.2.4 shows the different regions of an A-O system. 


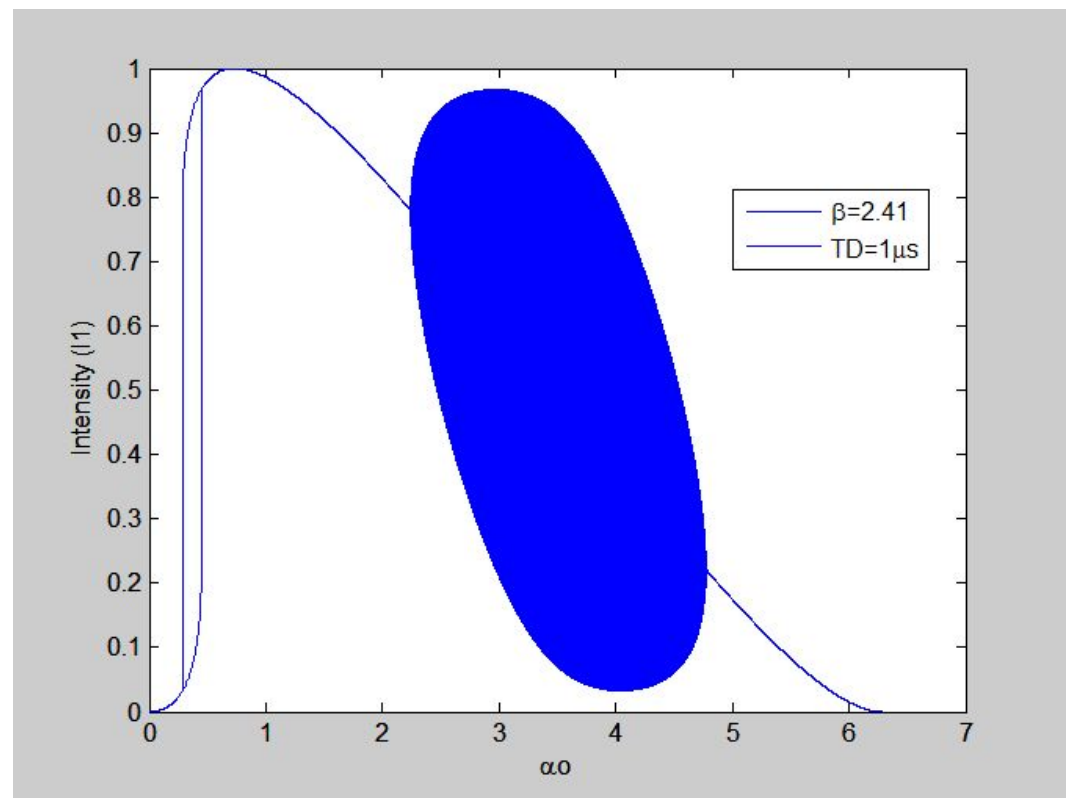

Fig.2.4. Different states of chaos.

Fig.2.4 shows different regions on the intensity vs bias input graph. Fig.2.4 indicates the lower monostable region where the first-order intensity has only one stable value for $\hat{\alpha}_{0}$ bias input. This is followed by the bistable region where the first-order intensity has two stable values for any single value of bias input. The system again enters a monostable region called the upper monostable region where the first-order intensity assumes a single value. Then the system enters chaos and undergoes multiple random oscillations. From the figure it is evident that the chaos starts after a particular (threshold) value of $\hat{\alpha}_{0}$ bias called $\hat{\alpha} \quad$ Th $\quad$ (after $\hat{\alpha}_{0}=2$ from Fig.2.4).

When we increase feedback gain $\widetilde{\beta}$ to 3 , the resulting NLD is as shown in Fig.2.5. 


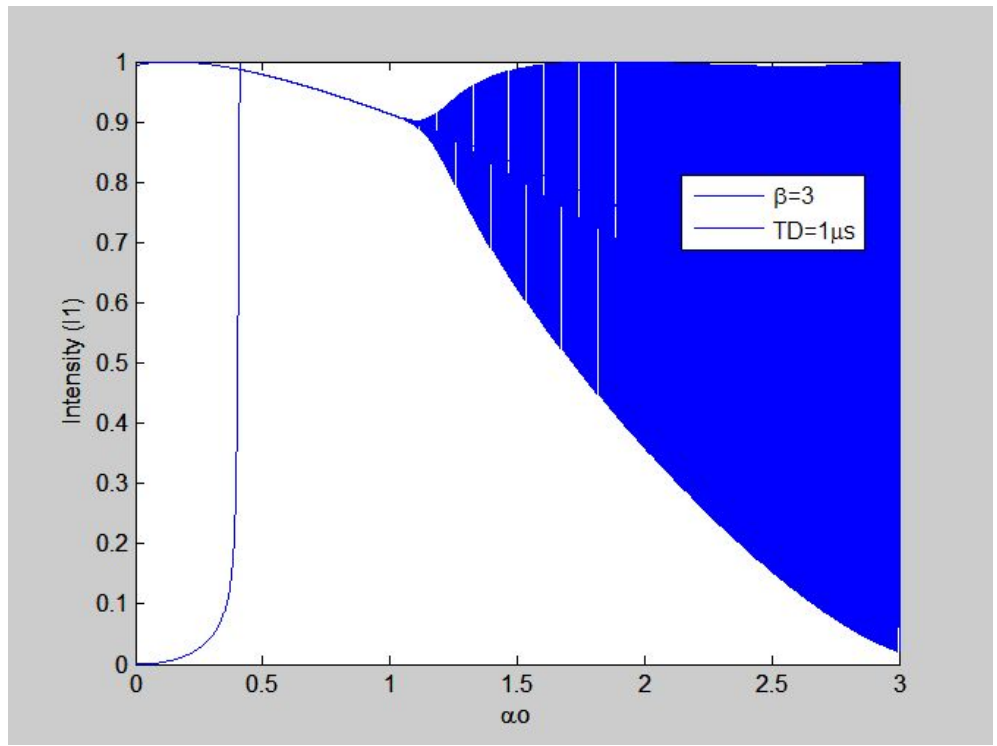

Fig.2.5. Chaos of feedback gain $\widetilde{\beta}$ is 3 .

Fig.2.5 shows certain differences from Fig.2.4. The lower monostable region has almost disappeared. The bistable region where the first order intensity has two stable values for any single value of bias input has become wider. The system again enters the upper monostable region where the first order intensity assumes a single value. Then the system enters chaos and undergoes mutiple random oscillations earlier than Fig 2.4. This shows that a change of feedback gain $\widetilde{\beta}$ leads to significant changes in the HAOF system.

As was seen from Fig.2.4, chaos starts after a particular value of $\hat{\alpha}_{0}$ bias called $\hat{\alpha}_{T h}$ Setting $I_{i n c}=1, I_{1}(0)=0$, and $\widetilde{\beta}=3$, time delay $(T D)$ is $1 \mu s$, and increasing $\hat{\alpha}_{0}$ bias by different values, we obtain the following five figures. 


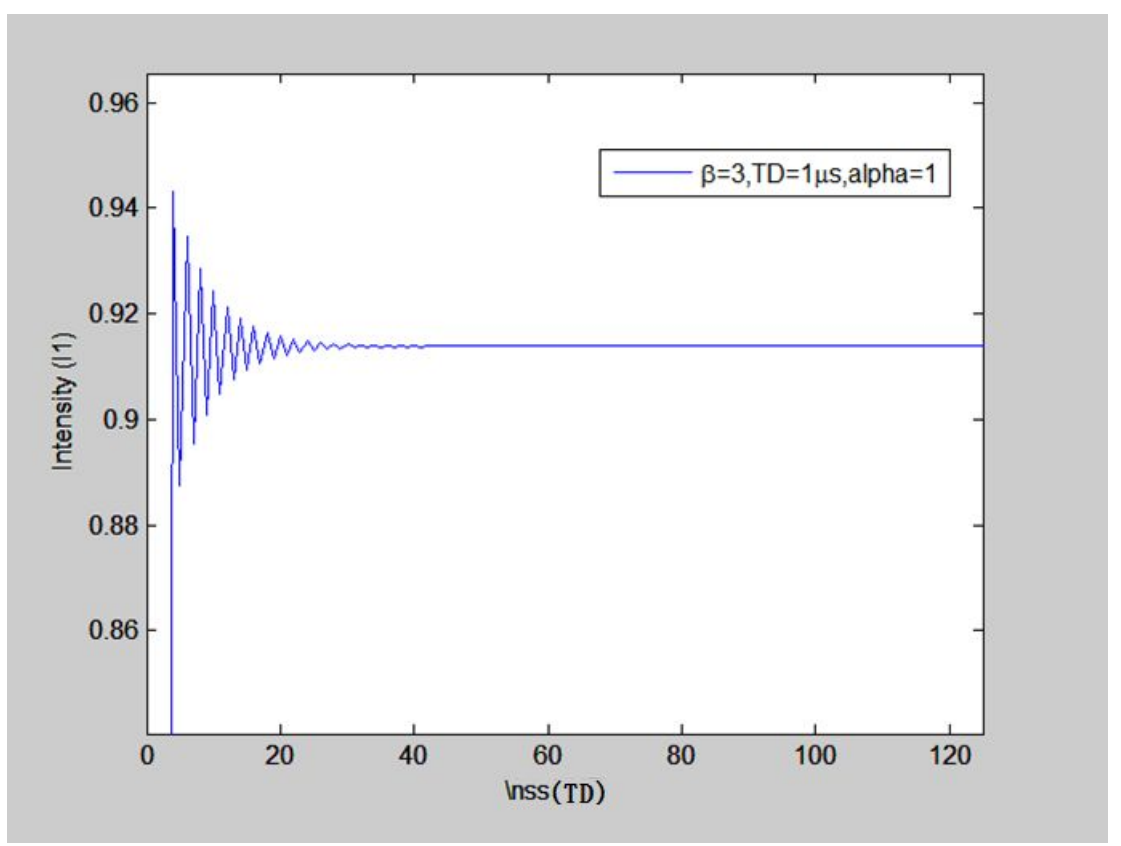

Fig.2.6. Chaotic Oscillations for $\hat{\alpha}_{0}=1$

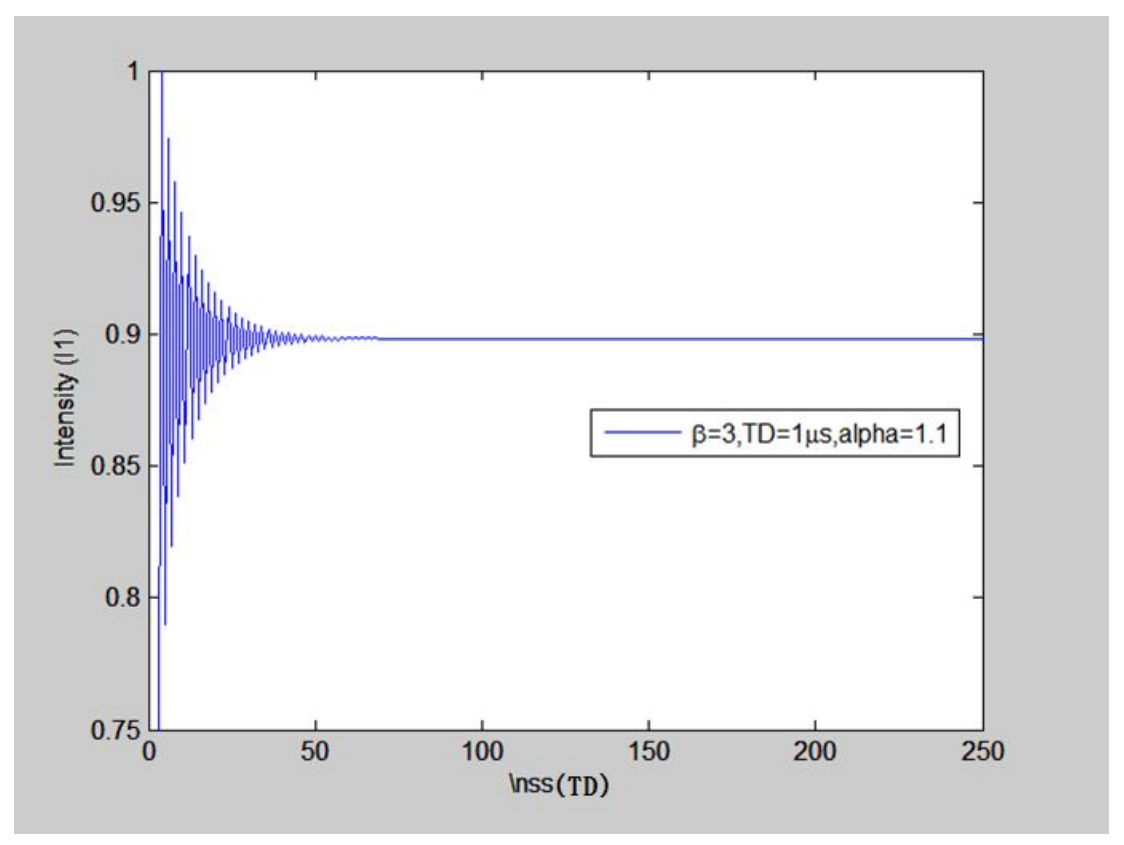

Fig.2.7. Chaotic Oscillations for $\hat{\alpha}_{0}=1.1$ 


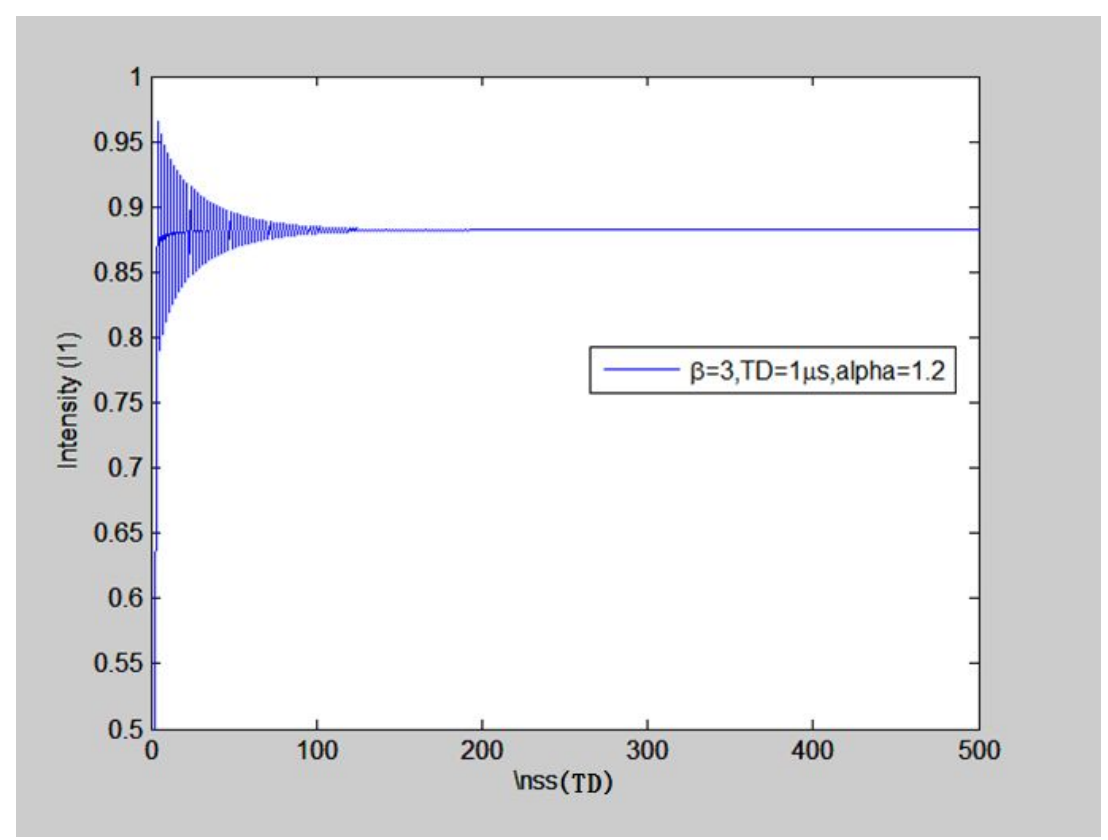

Fig.2.8. Chaotic Oscillations for $\hat{\alpha}_{0}=1.2$

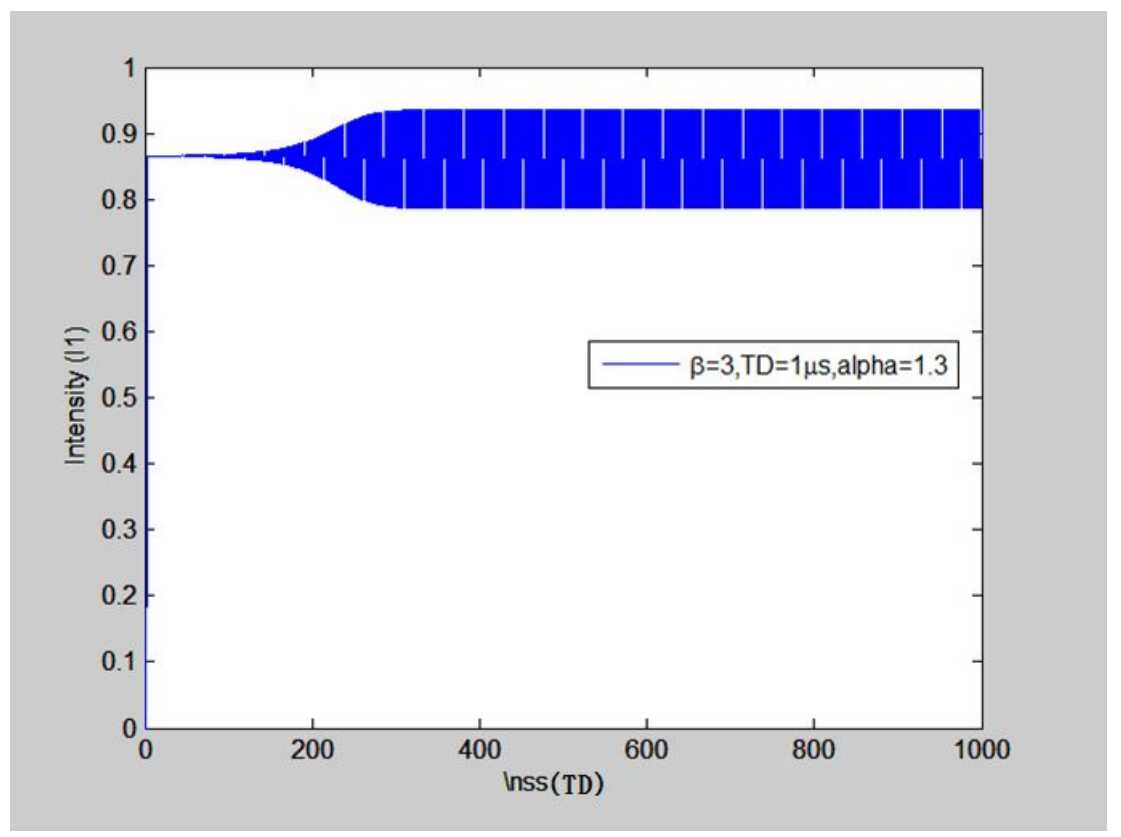

Fig.2.9. Chaotic Oscillations for $\hat{\alpha}_{0}=1.3$ 


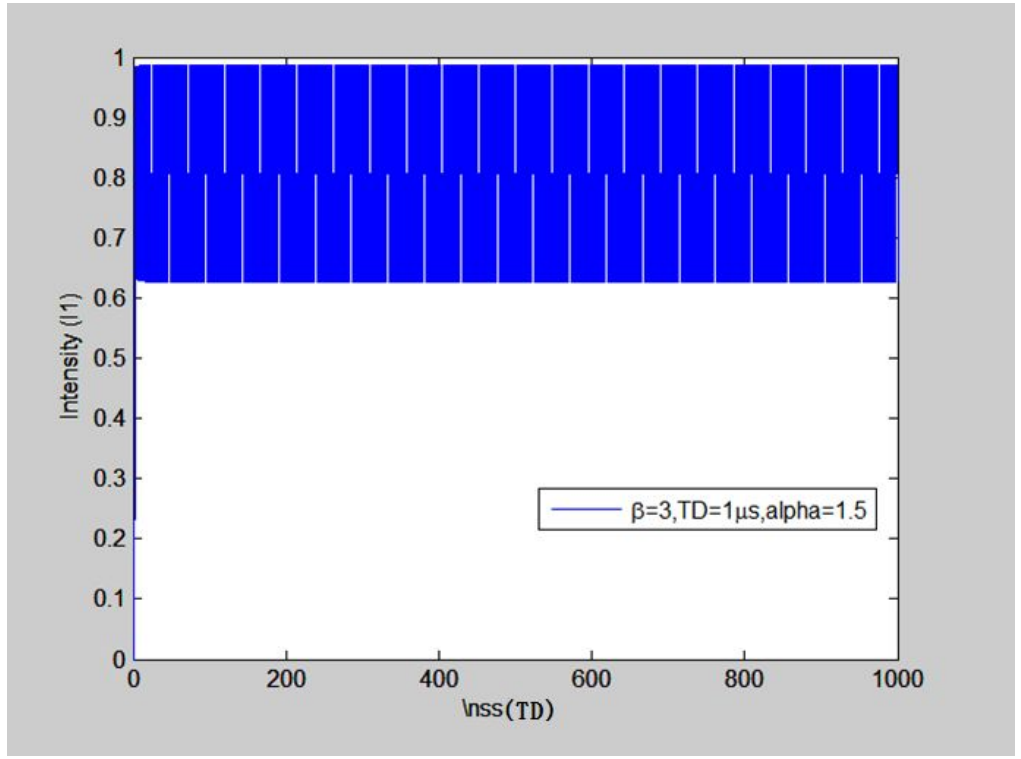

Fig.2.10. Chaotic oscillations for $\hat{\alpha}_{0}=1.5$.

From the above figures, we observe certain important characteristics. First, we find that the closed-loop system reaches a stable point in the NLD within different values of "settling time" for a range of values of $\hat{\alpha}_{0}$.Thus, we find that in Figs.2.6-2.8, the NLD settles into a fixed point within 42, 65 and 130 times the time delay (TD) for bias voltages of $\hat{\alpha}_{0}$ of $1,1.1$ and 1.2 respectively. When $\hat{\alpha}_{0}$ is increased to 1.3 , the system clearly enters chaos and there is no settling time as seen from Fig.2.9. When $\hat{\alpha}_{0}$ is increased to 1.5, the onset of chaos extends practically from $\mathrm{t}=0$.

\subsection{Examination of Dynamical Behavior Based on Bifurcation Maps}

Fig.2.11 shows the oscillations of a chaos wave when its feedback gain $\widetilde{\beta}$ is 3 and $\hat{\alpha}_{0}$ bias is 3.5 for a $\mathrm{TD}=1 \mu \mathrm{s}$. Fig.2.12 shows a snapshot of Fig.2.5 that shows the detailed oscillations. 


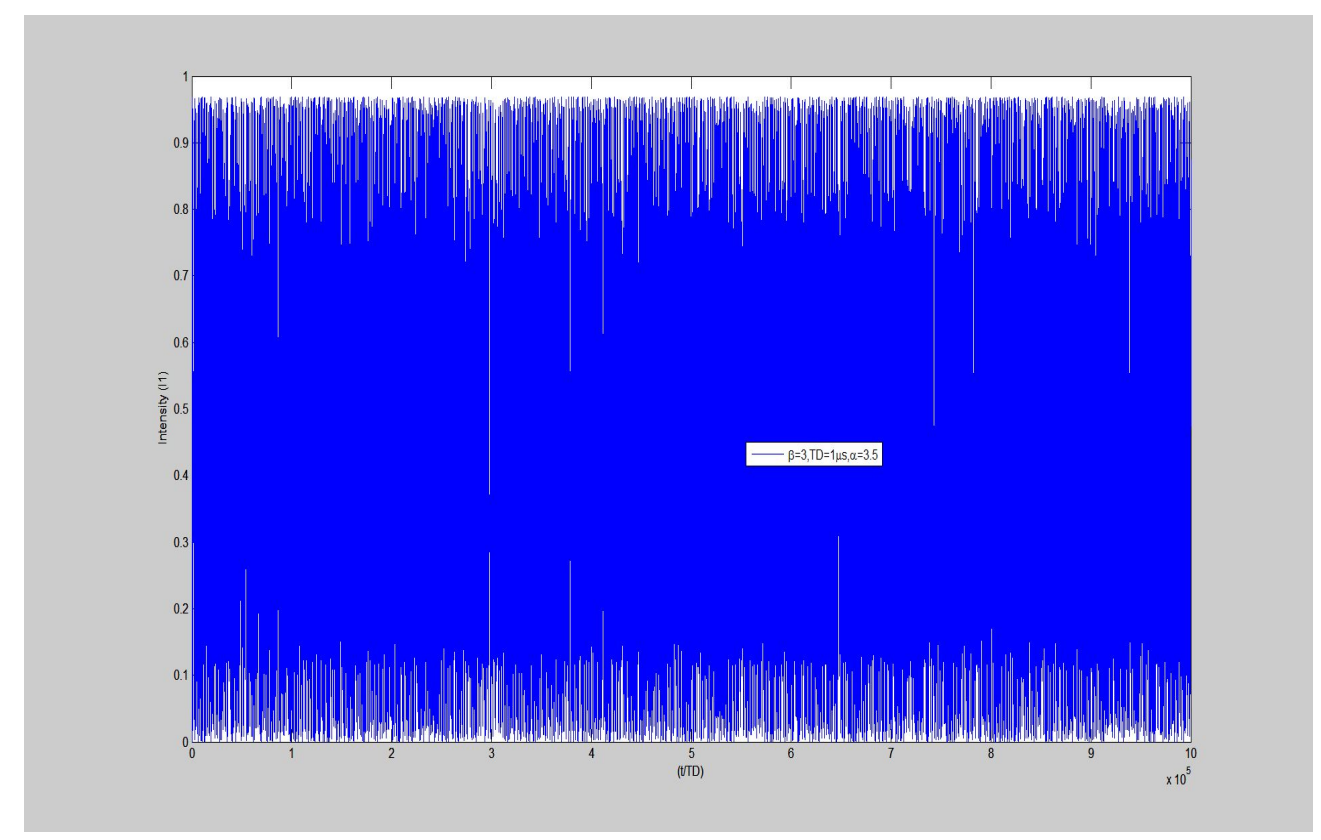

Fig.2.11. Chaos frequency of $\mathrm{t}=10^{6} \mathrm{TD}=1 \mathrm{~s}(\mathrm{TD}=1 \mu \mathrm{s})$

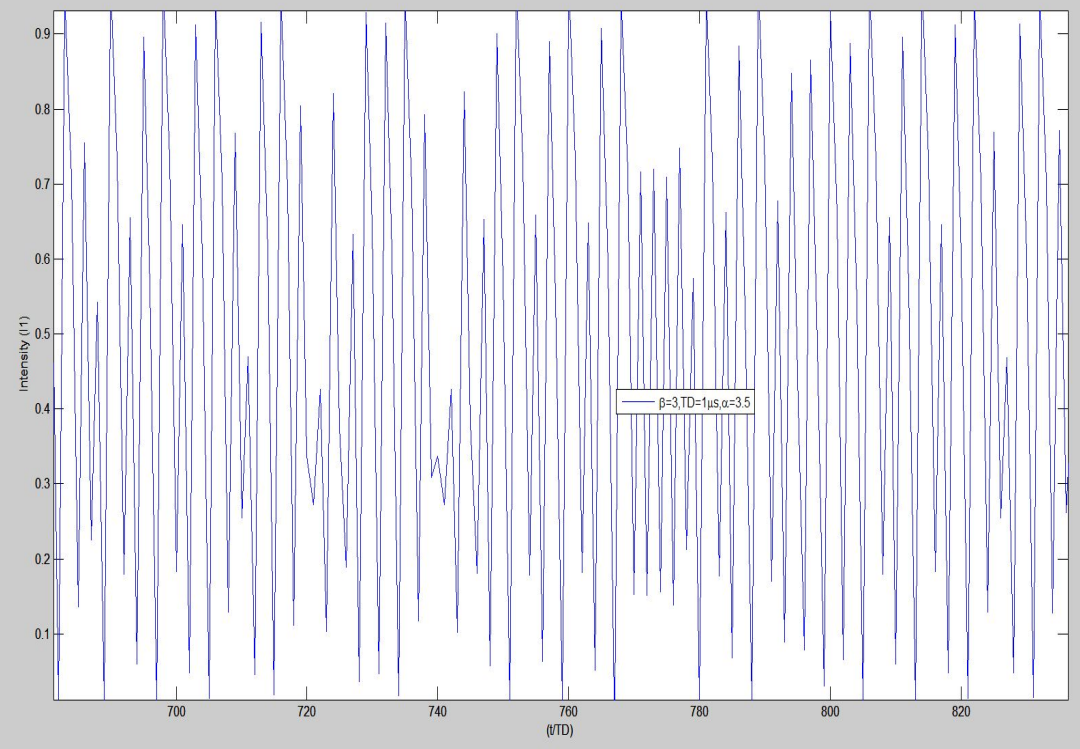

Fig.2.12. Snapshot chaos frequency of $\mathrm{t}=10^{6} \mathrm{TD}=1 \mathrm{~s}(\mathrm{TD}=1 \mu \mathrm{s})$ 


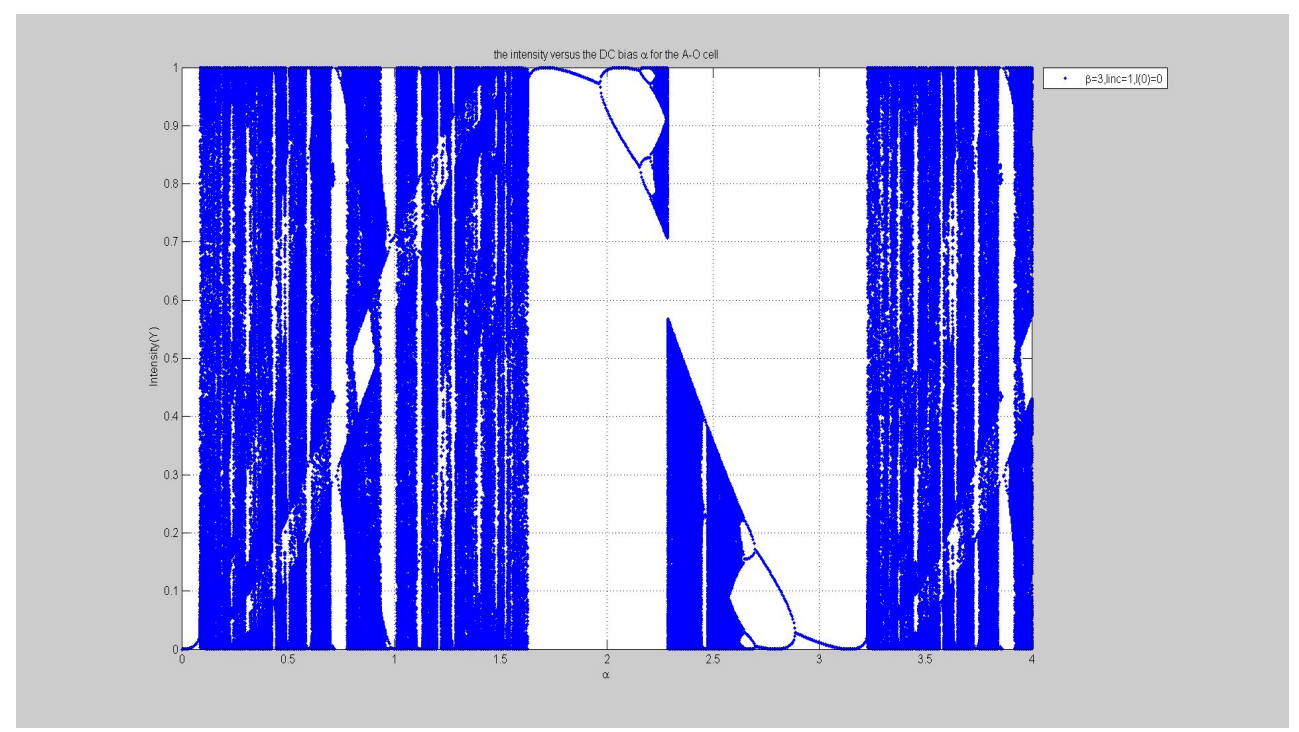

Fig.2.13. The bifurcation map of $I_{i n c}=1, I_{1}(0)=0$, and $\tilde{\beta}=3$

As shown in Fig.2.13, the blue part is the passband which means it has chaos and the white part is the stopband which means it has no chaos. We observe from Fig.2.11 that for $\hat{\alpha}_{0}=3.5$, there are chaotic oscillations versus time. In Fig.2.13, the bifurcation map shows a passband at $\hat{\alpha}_{0}=3.5$, thereby confirming that the system is in chaos. Thus, the results are compatible. As a sidelight, we observe that the extended stopband in Fig.2.13 is reminiscent of the Northwestern University logo. 


\section{CHAPTER 3}

\section{ACOUSTO-OPTIC CHAOS : A SIMULATION STUDY OF GENERAL CHARACTERISTICS FOR VARIABLE $\widetilde{\beta}(t)$}

\subsection{Approach}

The system shown in Fig.2.3 represented the standard Bragg cell transmitter with feedback. We next consider the case where instead of capturing all the incident light, the photo detector happens to have a variable aperture which may open and close with time. Different intensities of the first-order light would then be captured by the photo detector; this situation is somewhat comparable to having different photocurrents flowing in the feedback loop which might be equivalently described by having a fixed aperture with a variable feedback gain $\widetilde{\beta}(t)$ as Fig.3.1 shown.

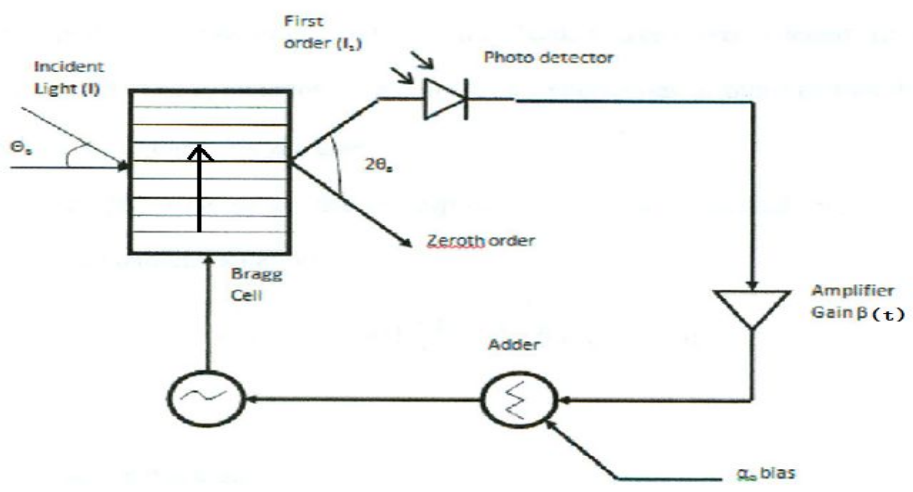

Fig.3.1. A-O Modulator with RF bias under variable feedback gain

In the initial tests, we set a variable feedback gain $\widetilde{\beta}(t)$ increasing with time as in Fig. 3.2 
in order to examine its NLD. Several trials indicated that the rate of rise of $\widetilde{\beta}(t)$ is critical in the closed-loop NLD of the system. If this rate is too small, there is no difference from the case of constant feedback gain $\widetilde{\beta}$. On the other hand, if it is too fast, the system may not enter chaos at all. Hence, as shown in Figs.2.6-2.10, shown we set the total duration of the variable feedback gain $\widetilde{\beta}(t)$ rising as a ramp to $10000 \mathrm{TD}$, within which the gain increase from 2.8 to 3.2 .

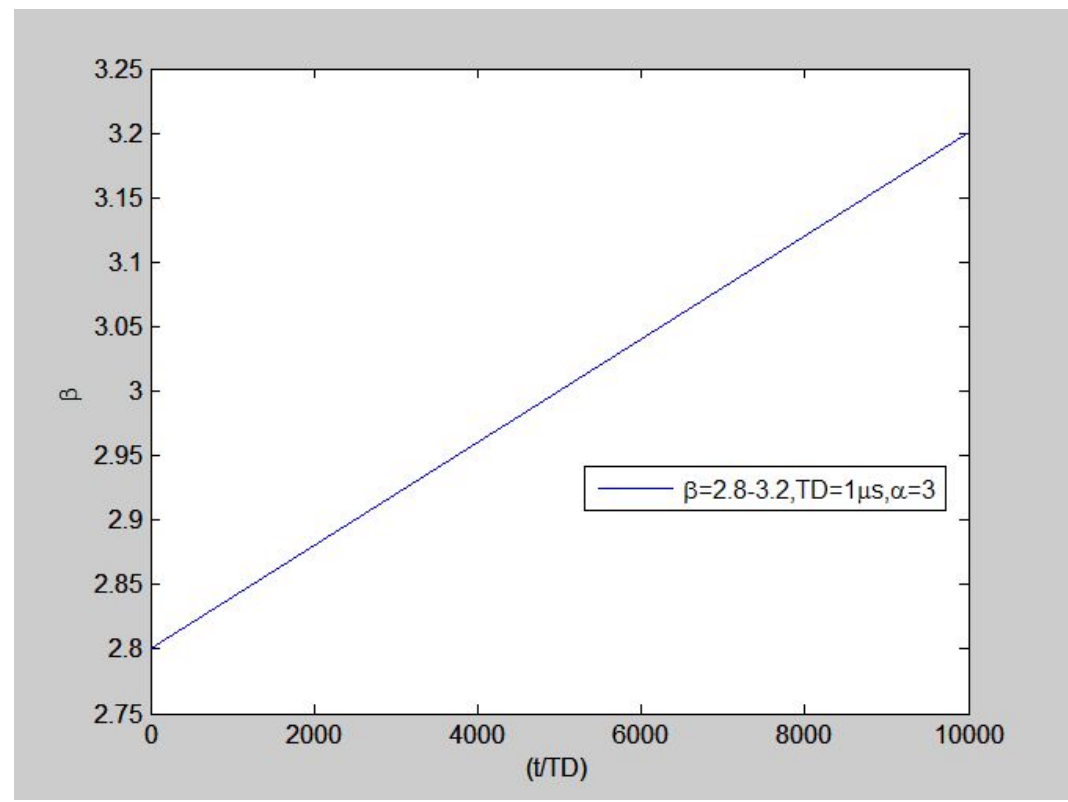

Fig.3.2. Feedback gain $\widetilde{\beta}(t)$ increase by time 


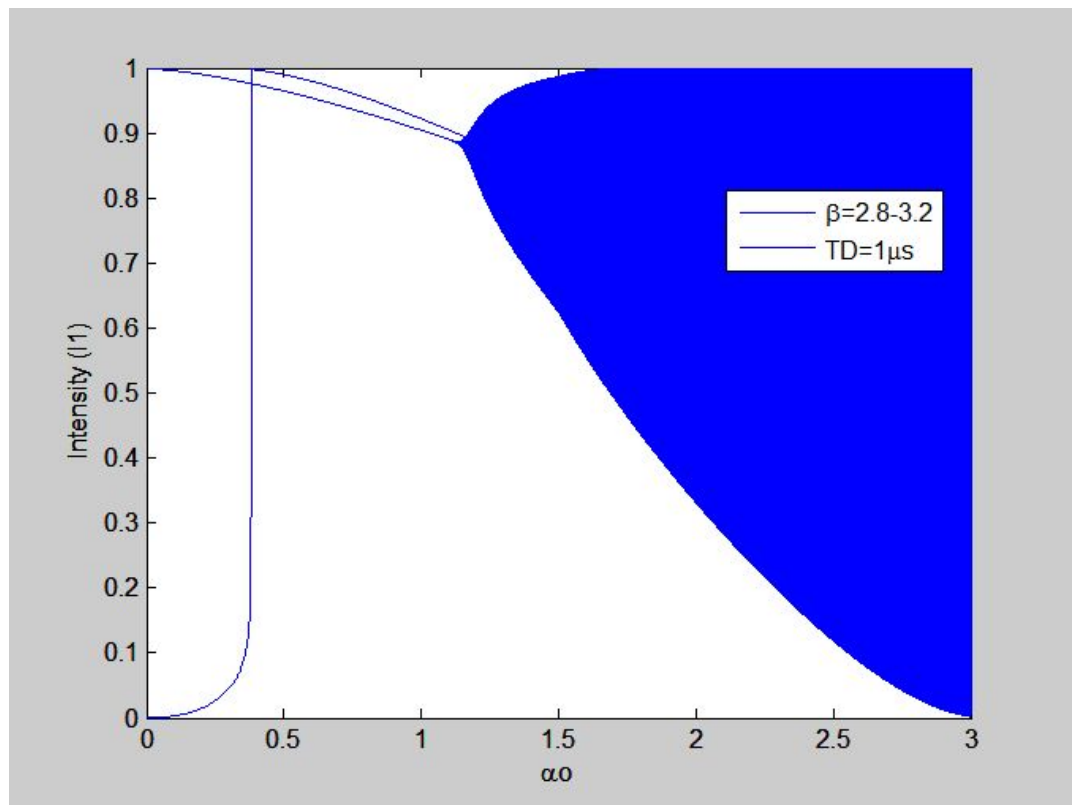

Fig,3.3. Chaos of variable feedback gain $\widetilde{\beta}(t)$

Fig.3.3 shows that the lower monostable region almost disappears compared with what was seen

in Fig.2.5. The bistable region where the first order intensity has two stable values for any single value of the bias input is wider. Then the system enters chaos and undergoes multiple random oscillations similar to Fig.2.5. This shows that a variable feedback gain $\widetilde{\beta}(t)$ leads to changes in the A-O chaos characteristics. Fig.3.3 and Fig.2.5 clearly show the differences between the NLD of the HAOF system for fixed versus variable feedback gain $\widetilde{\beta}$.

\subsection{Display of Time Oscillations Under Variable Feedback Gain}

Fig.3.4 shows the oscillation of a chaos wave when its variable feedback gain $\widetilde{\beta}(t)$ and $\hat{\alpha}_{0}$ bias is 3.5 for a $\mathrm{TD}=1 \mu \mathrm{s}$. Fig.3.5 shows a snapshot of Fig.3.4 that shows the detailed oscillation. 


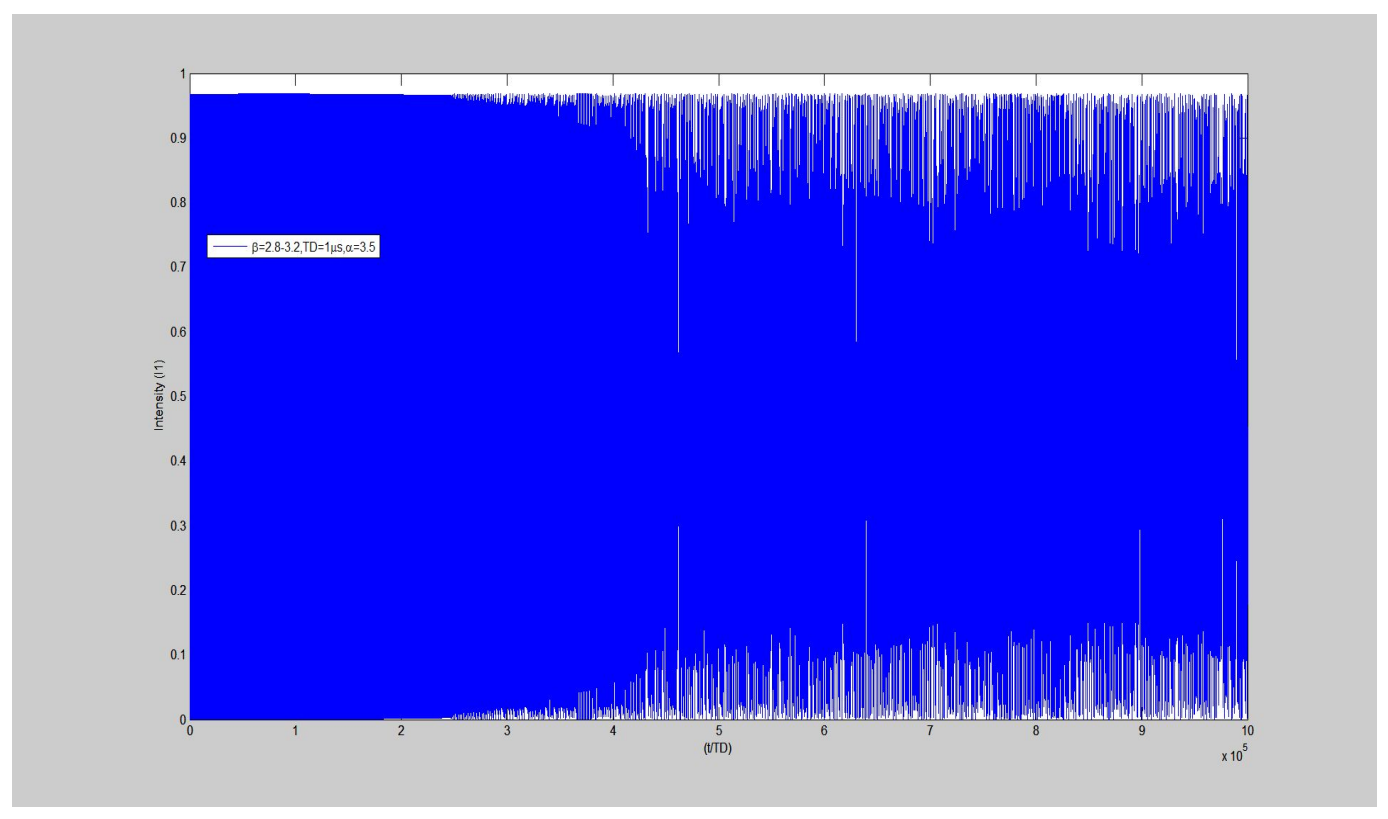

Fig.3.4. Variable feedback gain chaos frequency of $\mathrm{t}=10^{6} \mathrm{TD}=1 \mathrm{~s}(\mathrm{TD}=1 \mu \mathrm{s})$

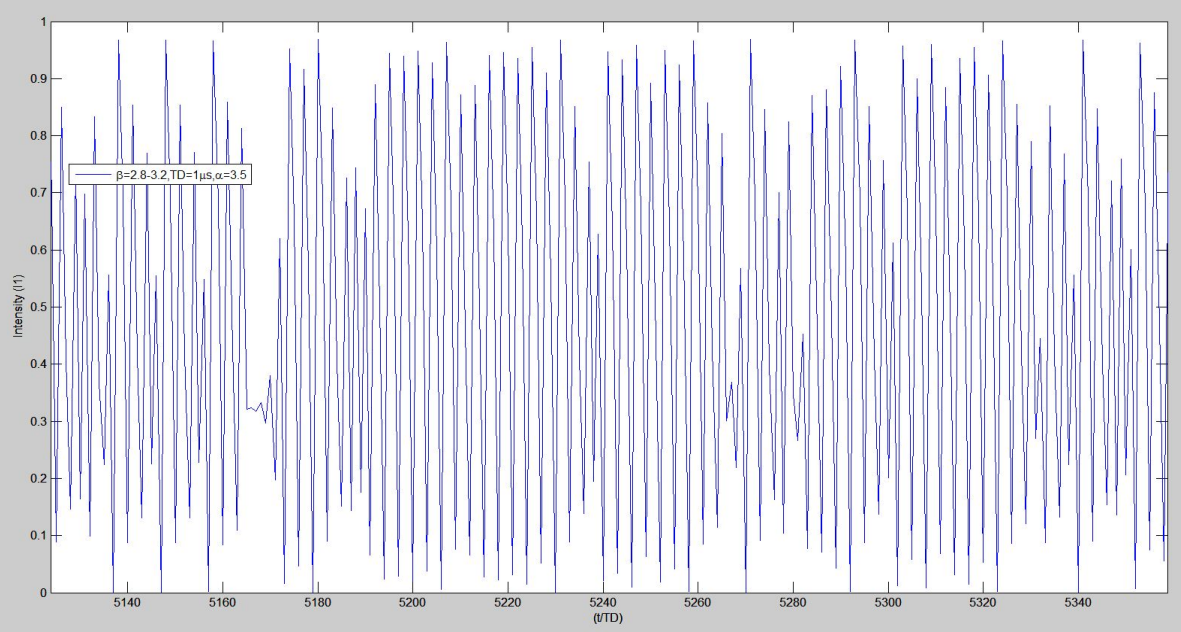

Fig.3.5. Snapshot variable feedback gain chaos frequency of $\mathrm{t}=10^{6} \mathrm{TD}=1 \mathrm{~s}(\mathrm{TD}=1 \mu \mathrm{s})$ 


\subsection{Examination of the Variable Feedback Gain Behavior Based on Bifurcation Maps}

Shown in the figures that follow are the bifurcation maps for different variable feedback gain ramps. Fig.3.6 is mostly like Fig.2.13 which is the bifurcation maps for a constant feedback gain (the white stopbands like the Northwestern logo). In Fig.3.8 the left pillar of the Northwestern logo has virtually disappeared (thus there is mostly a sea of blue passband, with only a narrow white pillar representing the stopband). Fig.3.7 shows characteristics somewhere between these two cases. These results indicate that a slow rise of $\widetilde{\beta}(t)$ over a wider amplitude range leads to a reduction of stopbands and a corresponding increase in the extent and bandwidth of the passbands. This would potentially increase the dynamic range of AC signals used in encryption applications.

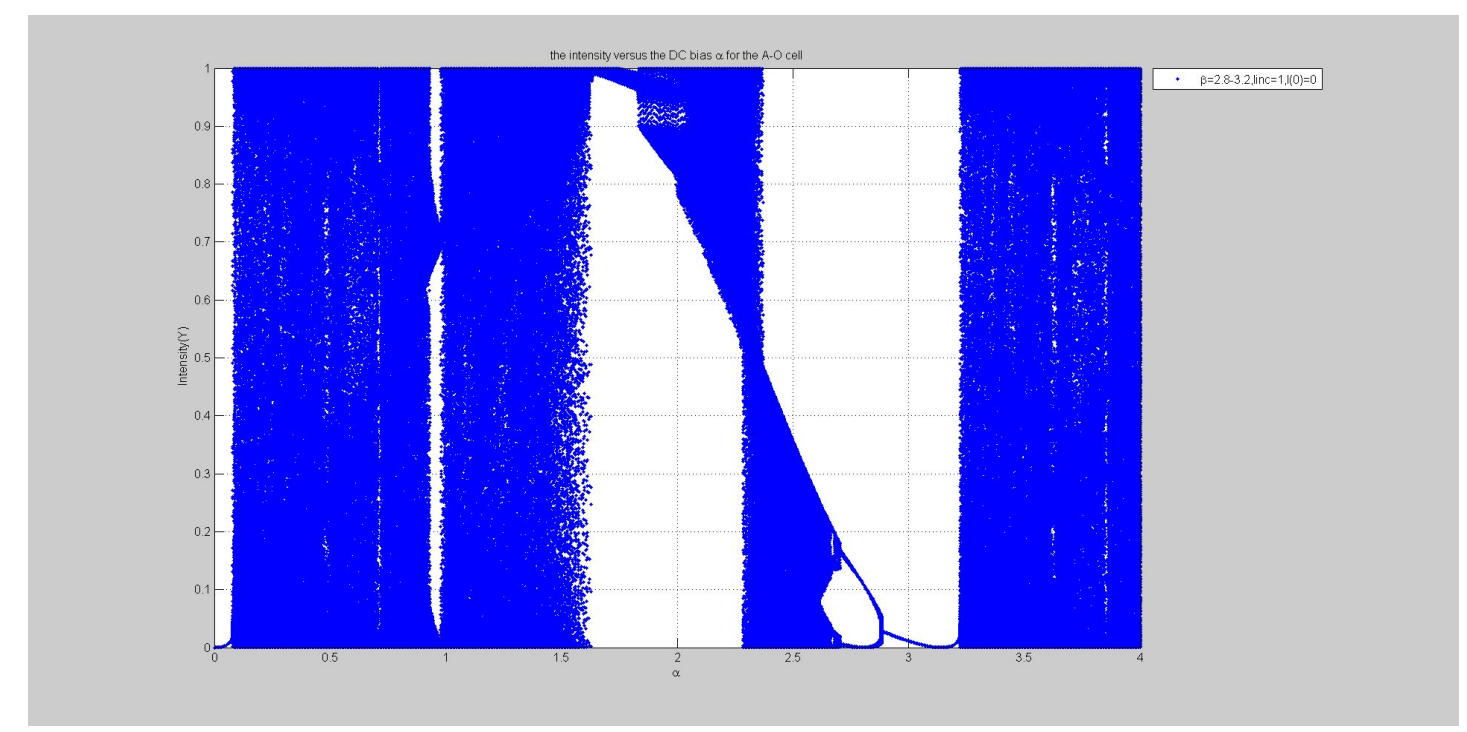

Fig.3.6. The bifurcation map of $I_{i n c}=1, I_{1}(0)=0$, and $\tilde{\beta}(t)=2.8-3.2$ 


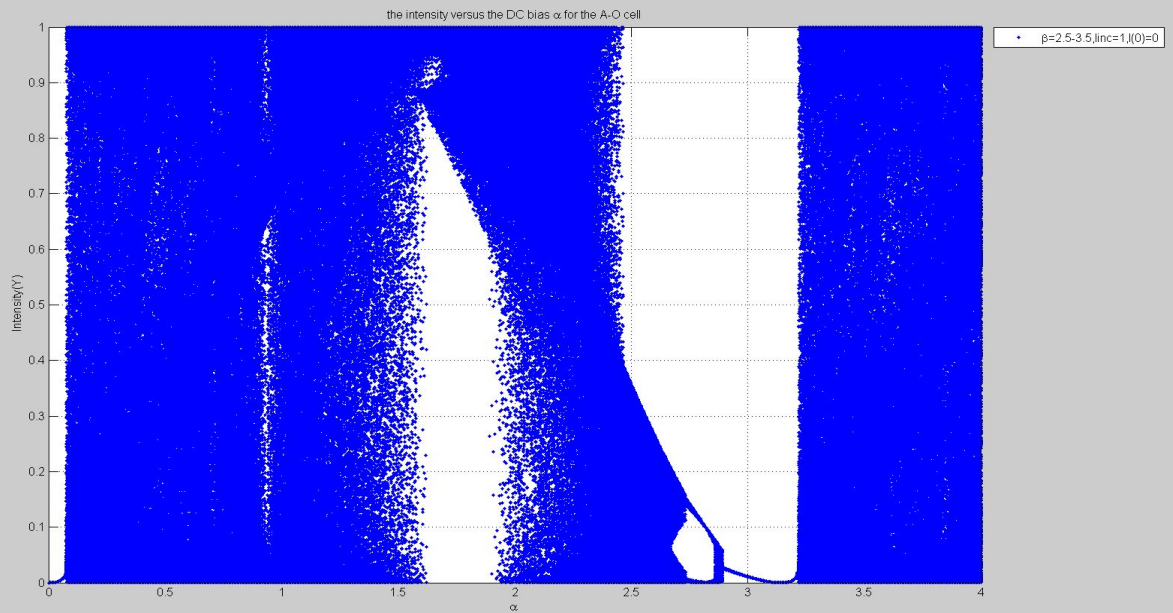

Fig.3.7. The bifurcation map of $I_{i n c}=1, I_{1}(0)=0$, and $\beta(t)=2.5-3.5$

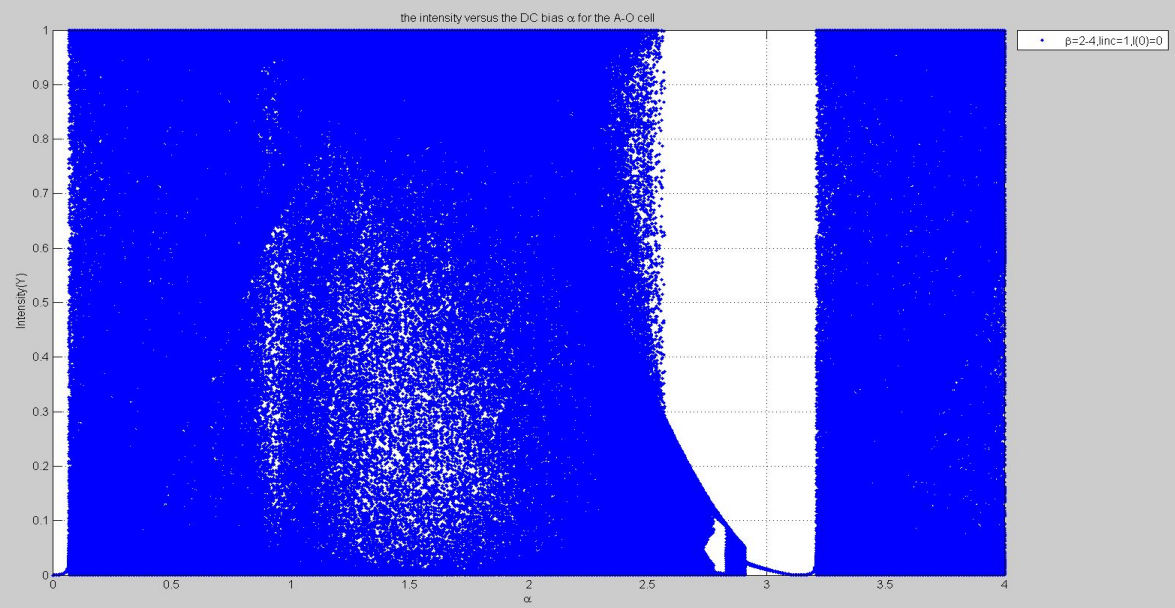

Fig.3.8. The bifurcation map of $I_{\text {inc }}=1, I_{1}(0)=0$, and $\tilde{\beta}(t)=2.0-4.0$ 


\section{CHAPTER 4}

\section{CHAOTIC SIGNAL ENCRYPTION AND DECRYPTION AND THE INFORMATION SECURITY OF THE VARIABLE FEEDBACK}

\section{GAIN}

\subsection{Background}

From many years there is a lot of research on providing high data security for many applications such as Banking, Defense security and So forth. In this chapter, we discuss one such method using chaos wave to encrypt and decrypt messages that provides a robust method for high data security.

Circuits or Optical systems that involve feed back and delay in their system have amplitudes or oscillations starting from stable state and oscillates between non periodic states. Although quantum cryptography is te termed as scheme for a data security, chaos encryption provides ultra-fast and most reliable method for this $[37,38]$. The system using chaos wave as carrier operates in real time and does not require complex algorithms to encode and decode messages at transmitter and receiver respectively. For example, encrypting and decrypting messages at the transmitter and receiver respectively using quantum cryptography involves the use of complex mathematical functions. All this involves complexity and time. Also in these schemes the key for decryption may itself be encrypted in the transmitted message, which might be at the risk of hacking if properly interpreted by 
some hacker. It also has no sort of relevance with the mathematical models used to encrypt the message.

Chaos may be referred to as distribution of random vibrations arising in nonlinear systems and may be defined by a set of non-linear differential equations. The chaos may occur in variety of fields ranging from physics, mathematics, biology and optics. Chaos enables resolution of many linear problems and its study had helped identify; various nonlinear phenomena. Development of modern high-speed computers has enabled the solution of complicated physical systems with high speed and accuracy.

\subsection{Signal Encryption in Chaos}

It was E.N Lorenz in 1963 who first found that in a set of three differential equations with non-periodic solutions, the solutions underwent irregular fluctuations, and were bounded but unstable [11]. But the term chaos for these observed irregular periodic oscillations was first termed by Poincare [12]. Encryption scheme in a chaotic system also follows the regular amplitude modulation (AM) for analog signals. It involves choosing a carrier close to chaos frequency $f_{c}$ and then encrypt the message on that carrier. The decoding at the receiver is similar to the heterodyne scheme used in the AM demodulation involves generating a local chaos carrier wave, then multiplying the local chaos wave with the received chaos + message signal and low pass filtering the product to filter out the high frequency carrier and retaining the low frequency message.

This may be mathematically described as [43] 


$$
s_{c h}(t)=[1+m s(t)] A_{c} \cos \left(2 \pi f_{c} t\right)+A_{c h} \cos \left(2 \pi f_{c h} t\right)
$$

Where $A_{c}$ and $A_{c h}$ are the amplitudes of the carrier and chaos respectively. Assuming $f_{c h}=f_{c}$ and by taking $A_{c h}+A_{c}$ as a common factor in eq.(4.1), we find:

$$
S_{c h}(t)=\left(A_{c}+A_{c h}\right)\left[1+\left(\frac{m A_{c}}{A_{c}+A_{c h}}\right) s(t)\right] \cos \left(2 \pi f_{c h} t\right)
$$

or, $\quad s_{c h}(t)=\tilde{A}_{c}[1+\tilde{m} s(t)] \cos \left(2 \pi f_{c h} t\right)$

$\tilde{A}_{c}$ and $\tilde{m}$ given eq.(4.3) are the effective chaos carrier amplitude and modulation index which are given by:

$$
\tilde{A}_{c}=A_{c}+A_{c h} ; \tilde{m}=\frac{m A_{c}}{A_{c}+A_{c h}}
$$

The equations 4.3, 4.4 define and describe how amplitude modulation carriers out and how a message may be modulated into a chaos carrier.

\subsection{Chaotic Encryption in HAOF Devices}

Ikeda in (1979) proved the concept of bistability and chaos in optical bistable devices 
theoretically using ring cavity [13]. This concept was later proven Gibbs in hybrid devices [14]. Optical oscillations and periodic disturbances were shown by Gibbs in hybrid optically bistable device with delay in the feedback loop and the results were in good agreement with the theoretical work done by Ikeda. The term "hybrid" in any system means that a part of the optical output is delayed electronically, amplified and fed back to the input of the bistable device.

In Bragg domain the amplitudes of the diffracted fields are governed by set of coupled differential equations. As explained in earlier chapters the first order light in Bragg regime is detected, amplified and fed back to the input. The feedback makes the system inherently nonlinear and complex in terms of its space-time dynamics. As mentioned in ref [18] the bistability and possible chaos depends on the choice of the feedback gain $\widetilde{\beta}$, input bias $\left(\hat{\alpha}_{0}\right)$ and the time delay (TD) in the loop. The chaotic encryption is accomplished by adding message signal to the dc bias input and the feedback input at the transmitter as shown in Fig.4.1

The receiver consists of a slave hybrid A-O circuit that generates a chaos wave that is in synchronization with the master hybrid A-O device used to generate chaos wave used for encrypting message at the transmitter. The locally generated chaos at the receiver is then multiplied with received chaos plus message signal, low pass filtered to extract the message signal. The synchronzation between the master chaos and local chaos is achieved by simple circuitry at the receiver. The circuit subtracts the slave chaos carrier from the master chaos carrier and the difference is added to the chaos carrier. This was designed by Mozdy in 1995 [39]. When the evolution of one of the coupled systems is unaltered by the coupling, the resulting configuration is called unidirectional coupling or 
drive-reponse coupling. On the contrary bidirectional coupling occurs when both systems are connected in such a way that they mutually influence each other's behavior [40-42].

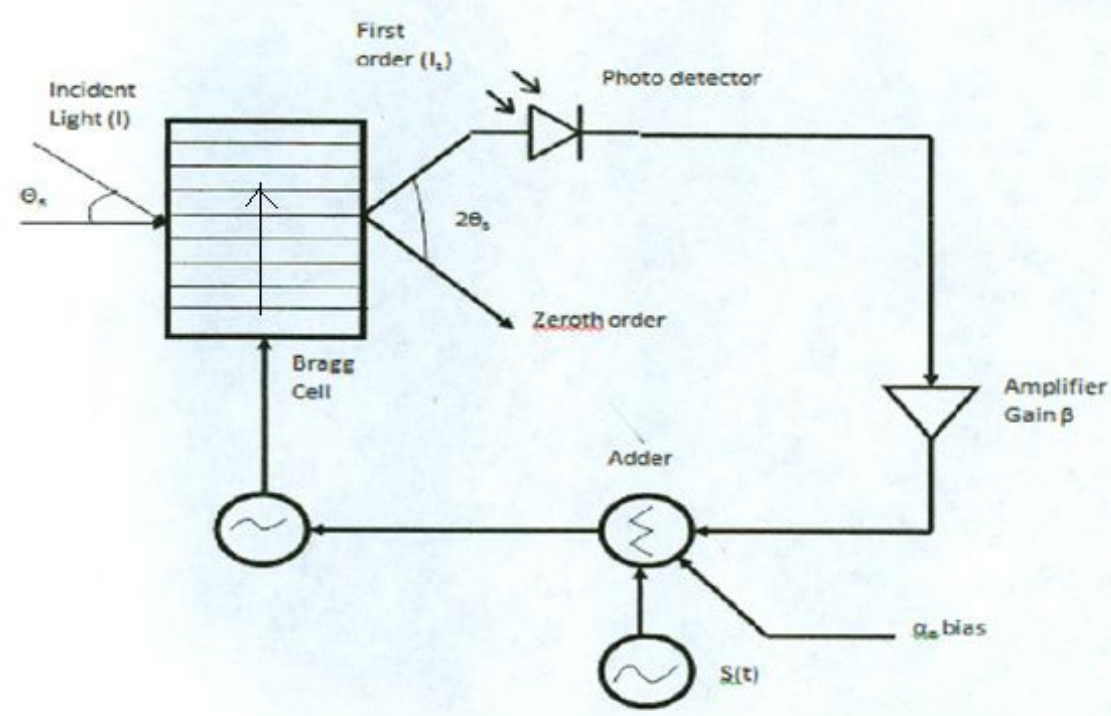

Fig.4.1. Chaotic encryption in a HAOF

\subsection{Choice of $\hat{\alpha}_{0}$ and Encryption}

As explained earlier encryption is done by adding ac message signal to the dc bias input $\hat{\alpha}_{0}$, at the adder which is then fed as input to the pezio electric transducer which generates the RF input for Bragg cell. Because of the the chaotic nature of the carrier the message signal is encrypted on the carrier and transmitted as shown in Fig.4.1.

The choice of $\hat{\alpha}_{0}$ is crucial to enable encryption on the chaotic carrier.From non linear analysis 
and bifurcation graphs of chaos vs different parameters that effect the chaos such as the feedback gain $\widetilde{\beta}$, input bias $\left(\hat{\alpha}_{0}\right)$ and the time delay (TD) performed by Monish Chatterjee and AI Saedi indicate different regions where chaos is observed [43] as shown in Fig.2.13.

From Fig.2.13 the blue area indicates the choice of $\hat{\alpha}_{0}$ where chaos is observed. From Fig 2.13, the sum of amplitudes of $\hat{a}_{a c}$ plus de bias $\hat{a}_{0}$ must lie within the blue block. All the encryption experiments in this paper have been performed around $\hat{a}_{0}=3.5$ and $\hat{a}_{a c}=0.25$ have been chosen so that at the adder, the sum of $\hat{a}_{a c}$ and $\hat{a}_{0}$ going from 0.25 to -0.25 always lies within in region around 3.5 , which is 3.25 and 3.75 . So that for the encryption $3.25<\hat{\alpha}_{\text {total }}<3.75$

\subsection{Receiver System and Decryption of Modulated Chaos}

The receiver scheme of the chaotic modulated signal is similar to that of an Amplitude demodulated wave. The receiver consists of a salve Bragg cell with only the DC bias input $a_{0}$ same as the master Bragg cell bias input driven into the RF transducer. The Bragg cell is so setup that it has the same $\hat{a}_{0}$, feedback gain $\widetilde{\beta}$ and the time delay (TD) as that of the original master Bragg cell of the transmitter. With no AC signal at the input the slave Bragg cell generates a chaos carrier similar to that used for encryption at the transmitter. The chaotic carrier at the transmitter and receiver must be in synchronization with each other. The received encrypted signal is first photo detected, then multiplied with locally generated chaotic carrier and then low pass filtered to remove high frequency component i.e the chaos carrier.Fig.4.2 shows the detailed diagram of the 
receiver.

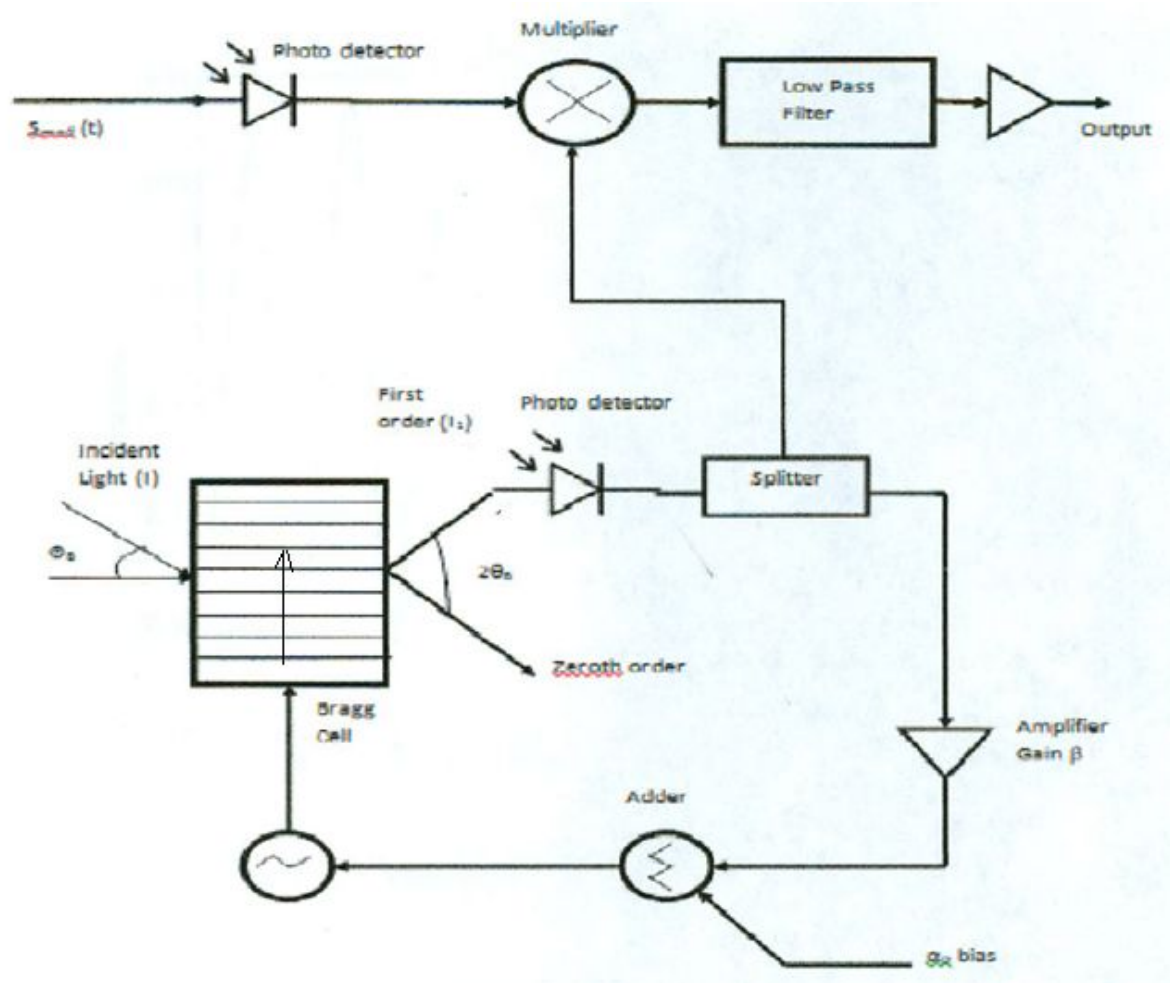

Fig.4.2. Chaotic decryption in a HAOF

\subsection{Chaotic Encryption and Decryption of the Constant Feedback Gain}

As shown in Fig.4.3, we set a input sinusoidal signal with frequency $500 \mathrm{~Hz}$, which is much smaller than the chaos frequency. After encryption and decryption with a constant feedback gain, the resulting recovered signal is shown in Fig.4.6, which shows that the filtered data is quite similar to the original signal. 


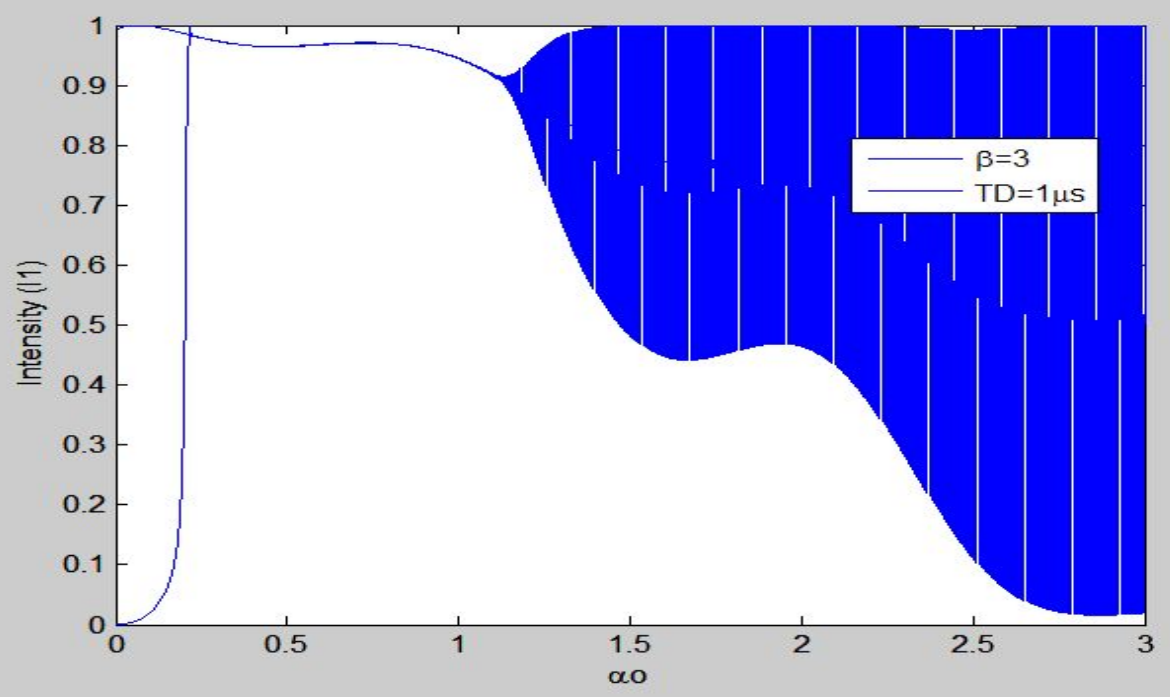

Fig 4.3 Chaos modulated sine wave of constant feedback gain $\widetilde{\beta}$

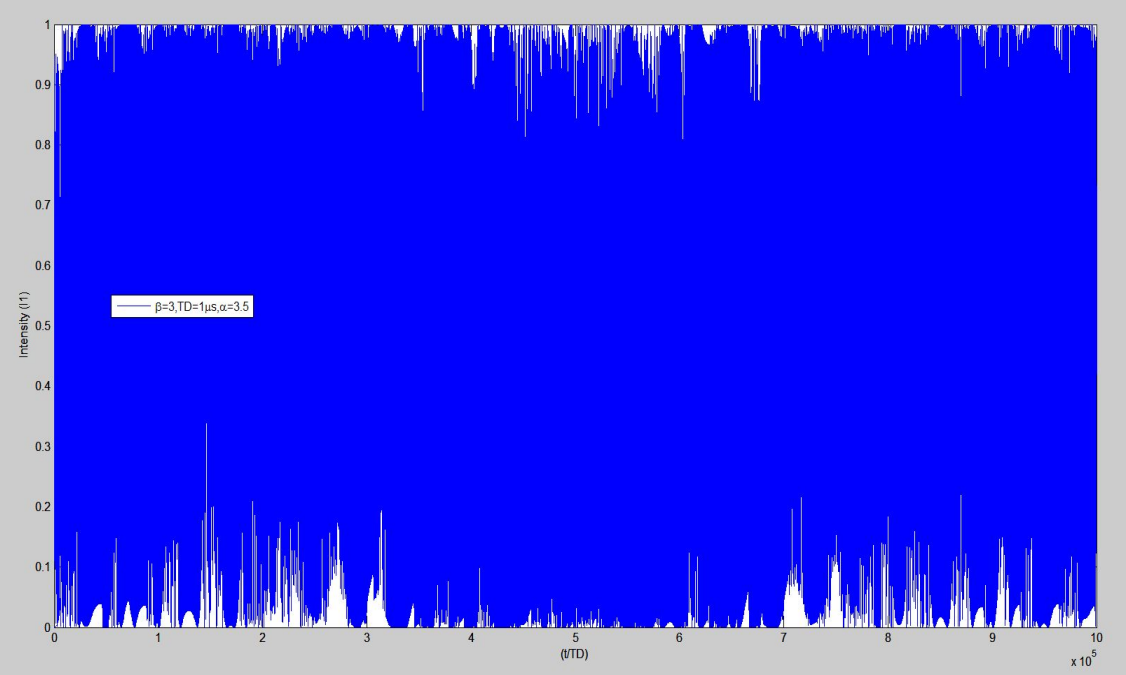

Fig.4.4. Constant feedback gain chaos modulated sine wave 


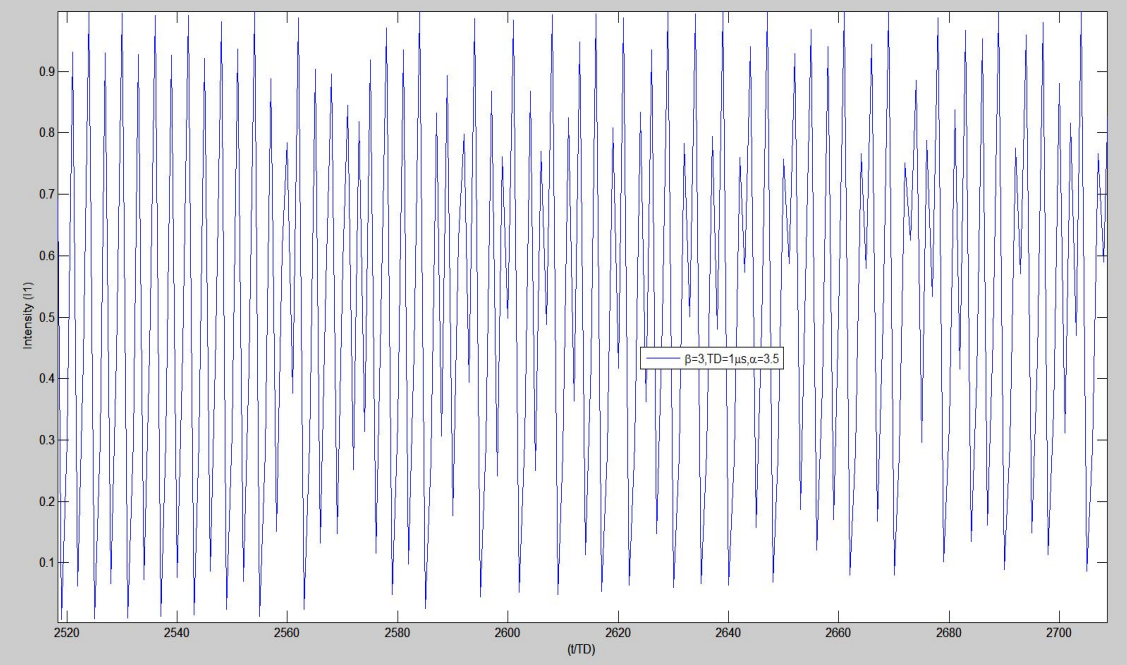

Fig.4.5. Snapshot constant feedback gain chaos modulated sine wave

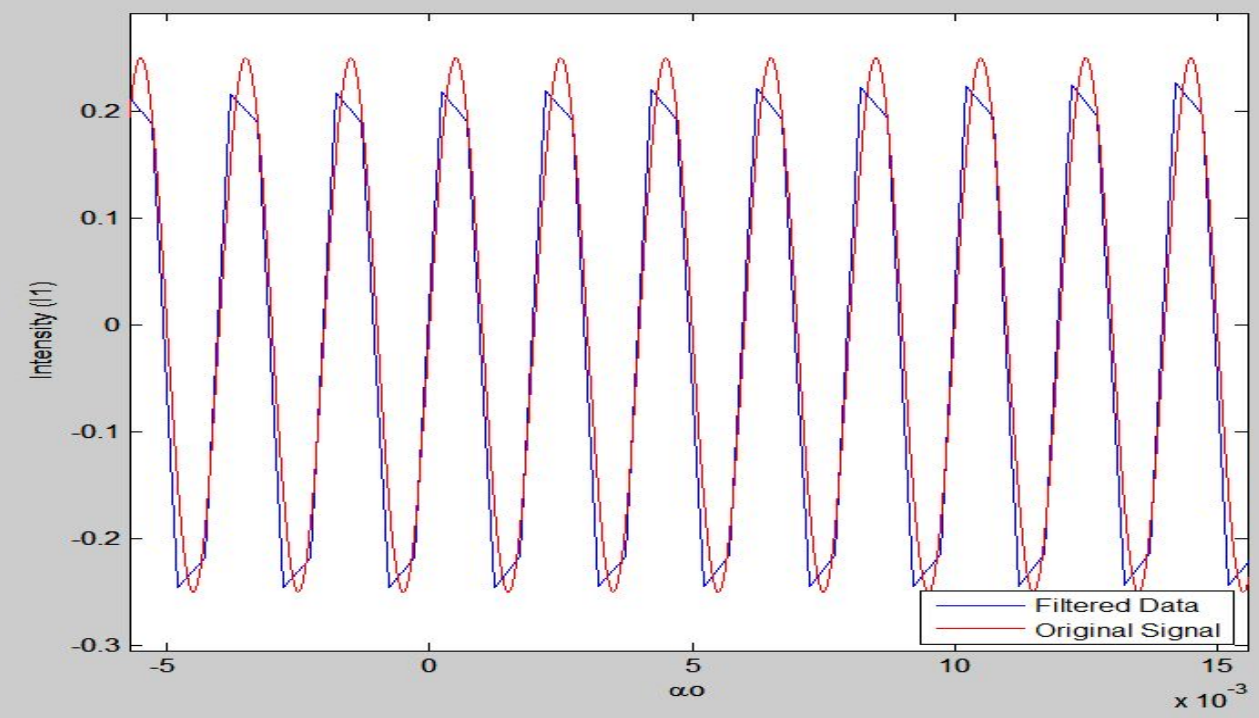

Fig.4.6. Comparison between filtered data and original signal for chaos modulation under constant feedback gain.

\subsection{Chaotic Encryption and Decryption of the Variable Feedback Gain}

As shown in Fig.4.7., we use a $500 \mathrm{~Hz}$ input sinusoidal signal, which is smaller than the rate of 
rise of the variable feedback gain and much smaller than the average chaos frequency. After encryption and decryption with the variable feedback gain, the resulting recovered output is shown in Fig.4.10., which shows filtered output data which is quite similar to the original signal.

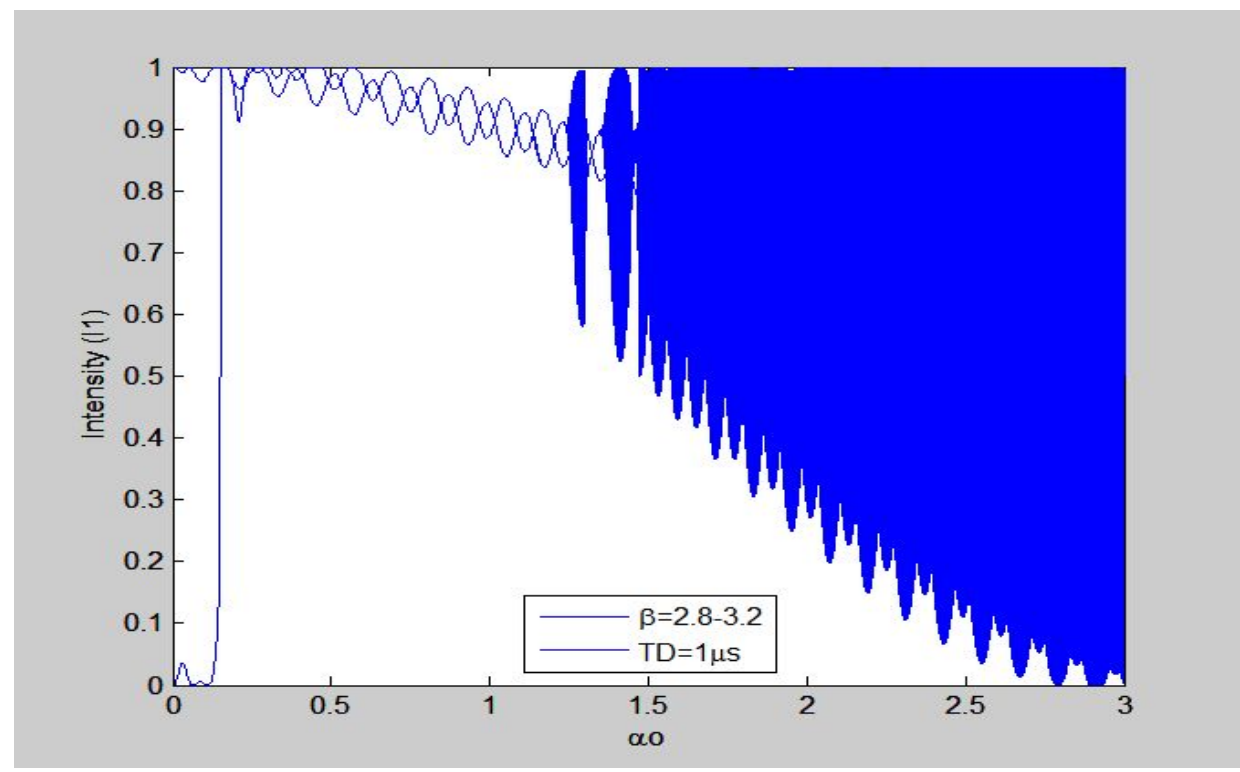

Fig.4.7. Chaos modulated sine wave of variable feedback gain $\widetilde{\beta}(t)$

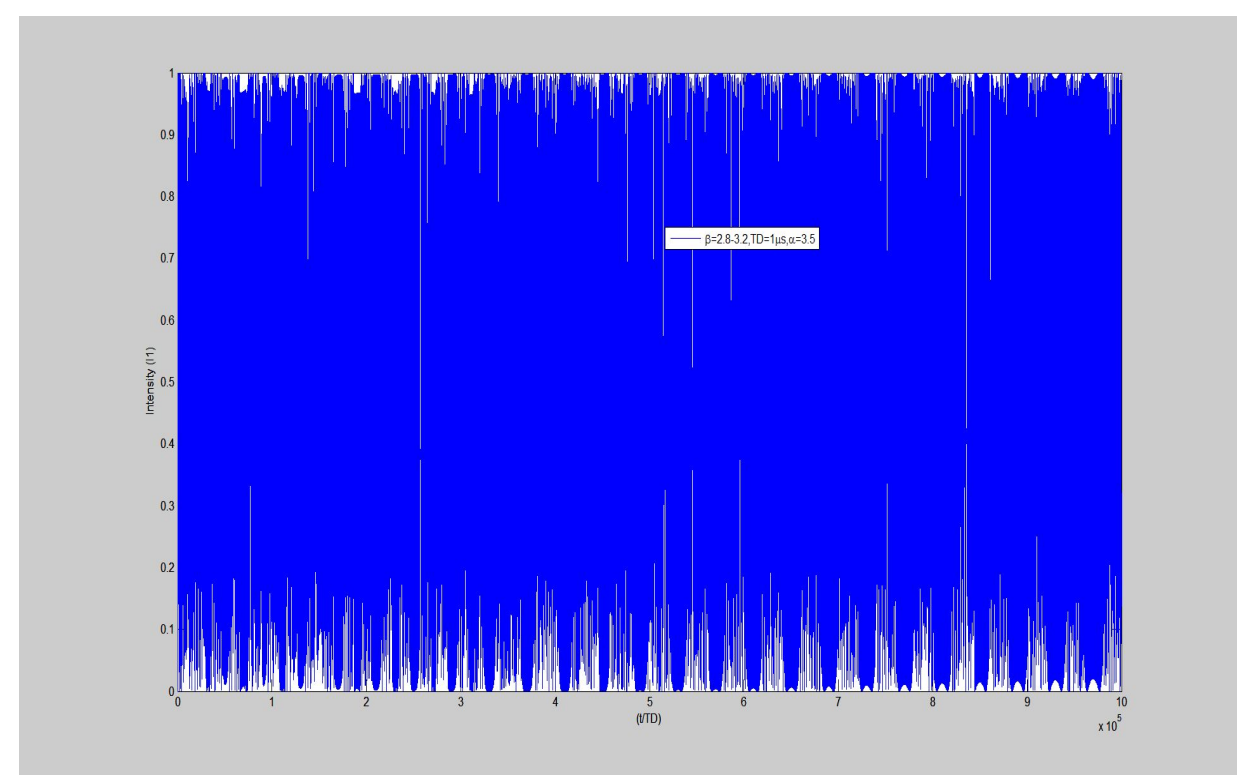

Fig.4.8. Variable feedback gain chaos modulated sine wave 


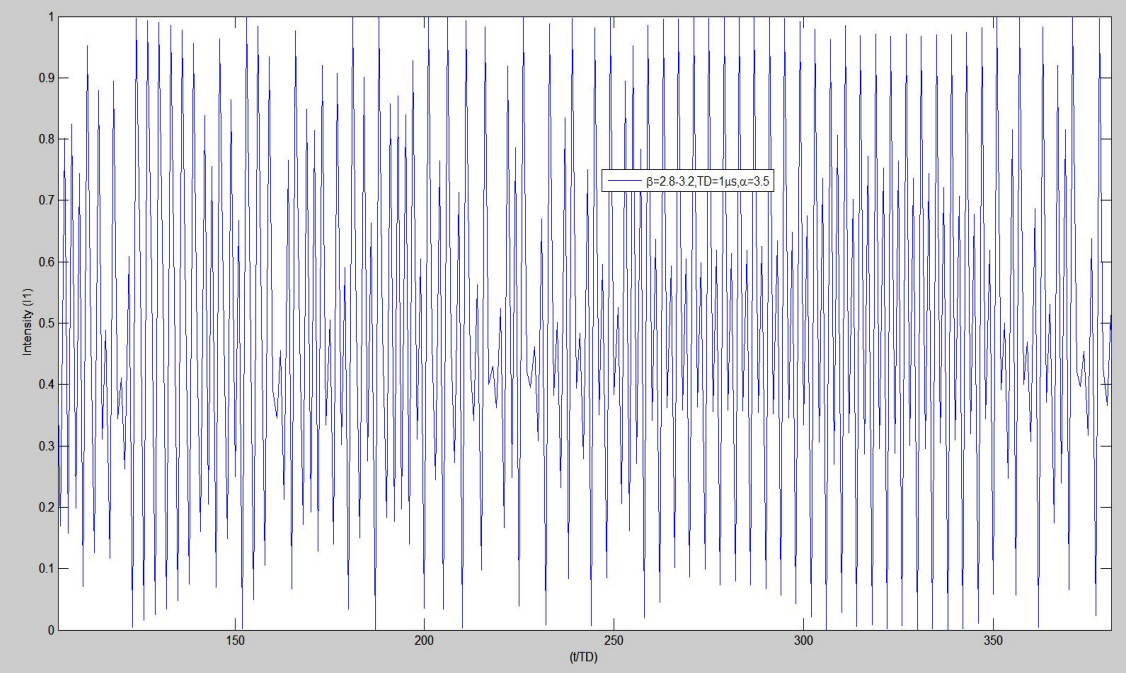

Fig.4.9. Snapshot variable feedback gain chaos modulated sine wave

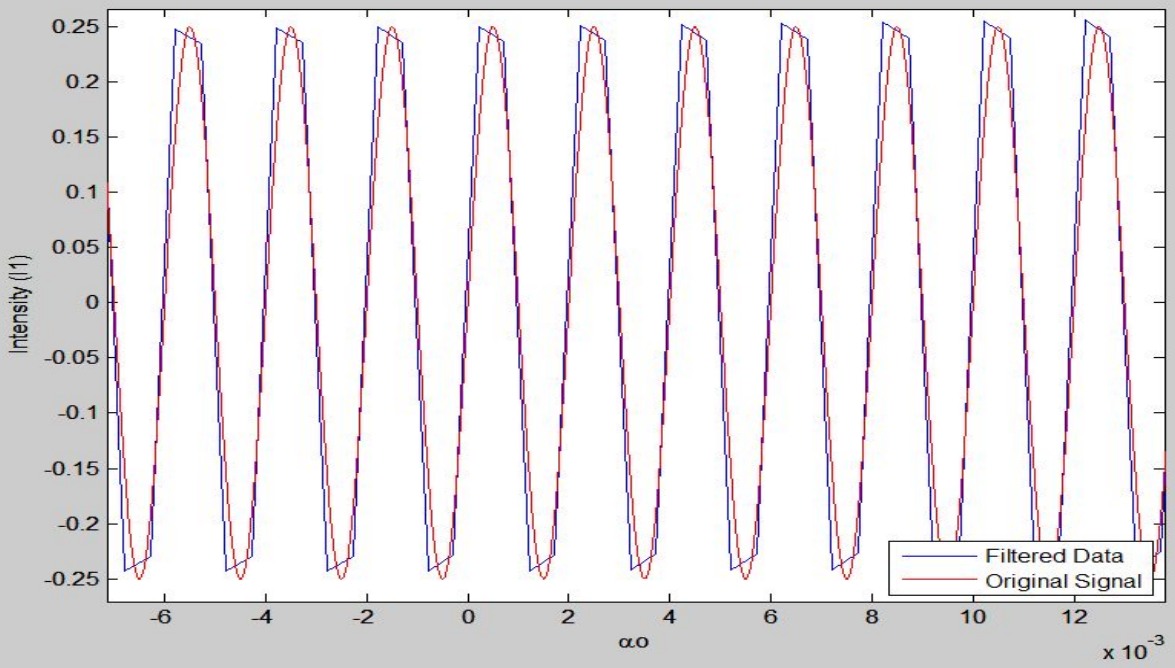

Fig.4.10. Comparison between filtered data and original signal for chaos under variable feedback gain.

From Figs. 4.6 and 4.10 it is clear that signal encryption and retrieval is possible even for variable feedback gain, as long as the gain does not change very rapidly, and the results are better if the gain varies over a wider amplitude within the allowed range of time. This research has not 
targeted performance evaluation of the variable gain problem along the lines of system robustness as typically tested via parameter mismatch between transmitter and receiver for the case of fixed feedback gain.

Low pass filter for variable feedback gain is much more special than fixed, the frequency of low pass filter should between modulated sine wave and feedback gain rising rate. Larger or smaller both can lead fail to decryption. It's a hard work for finding a suitable low pass filter to each HAOF system under a variable feedback gain. 


\section{CHAPTER 5}

\section{CONCLUSION}

This research focused on the optical characteristics of a hybrid A-O feedback device with variable gain. We have generated chaos and allied conditions corresponding to constant and variable feedback gain $\widetilde{\beta}(t)$, and studied in detail the NLD of the two systems. The generation of chaos has been studied for different parameters such as feedback gain $\widetilde{\beta}$, input bias $\left(\hat{\alpha}_{0}\right)$ and the time delay (TD). Additionally, the variable gain system has been designed for applications to signal encryption and decryption utilizing modulated chaos waves with uniform incident plane waves. The present work originated with the problem of a variable aperture opening relative to the first-order light. This potentially complex problem is simplified by assuming instead a variable feedback gain $(\widetilde{\beta}(\mathrm{t}))$, which leads to considerably different nonlinear dynamics. This document explores initially the simpler stages of this problem by examining the nonlinear dynamics versus the (DC) bias voltage for different variable- $\widetilde{\beta}$ conditions, including very slow and likewise fast rates of change of the gain with time in relation to the feedback delay. It is found that the response depends critically on the rate of rise of the feedback gain, and also that the resulting chaotic regimes are generally significantly different from those for fixed values of

$\widetilde{\beta}$. We use chaos under constant and variable feedback gain $\widetilde{\beta}(t)$ to encrypt messages which 
may be useful in information security applications. A receiver scheme has been developed using a second Bragg cell with matched bias parameters to generate local chaos followed by a low pass filter. This heterodyne system then extracts the securely transmitted message. It is generally observed that the chaotic system experiences more passband and stopband transitions for comparable system parameters relative to a fixed- $\widetilde{\beta}(t)$ problem. This research provides some insight into the NLD of variable feedback, and potentially holds out the promise that the resulting system when properly designed may offer higher system robustness, and also greater dynamic ranges for information under transmission.

\section{Future work:}

A - O networks have wide areas of application and research, and a quite a bit more may be studied in this area using chaos and the feedback system. As mentioned, further studies may not only include more complex chaos and feedback analysis, but such modifications that may lead improved robustness for the system and more versatile handling in terms of performance. Some such possibilities may include:

1) The chaos frequency may be extended into the optical range.

2) A video input may be encrypted with the chaos carrier and may be transmitted and received successfully at the receiver. Some work along this direction is already in progress.

3) More variations in the feedback gain profile may be further studied to derive deeper understanding of this complex physical system. 


\section{REFERENCES}

[1] B.-L. Hao, "Chaos," $11^{\text {st }}$ edition (World Scientific, Singapore, 1984).

[2] J.W.S. Rayleigh, "On convective currents in a horizontal layer of fluid when the higher temperature is on the underside," Phil. Mag. 32, 529-530 (1916).

[3] J.W.S. Rayleigh, "The Therory of Sound," $2^{\text {nd }}$ edition (Macmillan and Co.Ltd, New York, 1976).

[4] J.P. Gollub, T.O. Brunner, and B.G. Danly, "Periodicity and chaos in coupled nonlinear oscillators," Science 200, 48-50 (1978).

[5] J.P. Gollub, E. J. Romer, and J.E. Socolar, "Trajectory divergence for coupled relaxation oscillators: measurements and models,” J. Stat. Phys. 23, 321-325 (1980).

[6] P.S. Lindsay, "Period doubling and chaotic behavior in a driven harmonic oscillator," Phys. Rev. Lett. 47, 1349-1352 (1981).

[7] J.Perez, and C. Jeffries, "Effects of additive noise on a nonlinear oscillator exhibiting period-doubling and chaotic behavior," Phys. Rev. B26, 3460-3463 (1982).

[8] E.R. Hunt, "Comment on a driven nonlinear oscillator," Phys. Rev. Lett. 49, 1054-1056 (1982). 
[9] R.W. Rollins, and E.R. Hunt, "Exactly solvable model of physical system exhibiting universal chaotic behavior,” Phys. Rev. Lett. 49, 1295-1298 (1982).

[10] S.D. Brorson, D. Dewey, and P. S. Linsay, "Self-replicating attractor of a driven semiconductor oscillator,” Phys. Rev. A28, 1201-1206 (1983).

[11] E.N. Lorenz, "Determinnisitic non-periodic flow," J. Atoms. Sci. 20, 130-141 (1963).

[12] T.Y. Li and J.A. Yorke, "Period three implies chaos," Am. Math. Monthly 82, 985-992 (1975).

[13] K. Ikeda, "Multiple-valued stationary state and its instability of the transmitted light by a ring cavity system,” Opt. Comm. 30, 257-261(1979).

[14] H.M. Gibbs, F.A. Hopf, D.L. Kaplan and R.L Soemaker, "Observation of chaos in optical bistability," Phys, Rev. Lett. 46, 474-477 (1981).

[15] R.G. Harrison, W.J. Firth, C.A. Emshary and I.A. Alsaidi, "Observation of period doubling in all optical resonator containing NH3 gas,” Phys. Rev. Lett. 562-565 (1983).

[16] J.Chrostowski and C. Delisle, "Bistable piezoelectric Fabry-Perot interferometer" Can. J. Phys., 57, 1376-1379 (1979).

[17] J.Chrostowski and C. Delisle, "Bistable optical switching based on Bragg diffraction," Opt. Comm. 41, 71-74 (1982).

[18] J.Chrostowski, C. Delisle and R. Tremblay, "Oscillations in acousto-optic bistable device," Can. J.Phys., 61, 188-191 (1983).

[19] R.Valle, and C. Delise, "Route to chaos in an acousto-optic bistable device," Am. Phys. 
Society, 31, 2390-2396 (1985).

[20] L.Larger, J-P Goedgebuer, and F. Delmore, "Optical encryption system using hyperchaos generated by and F. Delmore, "Optical encryption system using hyperchaos generated by an optoelectronic wavelength oscillator,” Phy. Rev. 57, 6618-6624 (1998).

[21] C. Mirasso, R. Vicente, P. Colet, J. Mulet, and T. Perez, "Synchronization properties of chaotic semiconductor lasers and applications to encrypyion," C.R. Physique 5, 613-622 (2004).

[22] L. M. Pecora, and T.L. Carroll, "Synchronization in chaotic system," Phys. Rev. Lett. 64, 821-826 (1990).

[23] E.Ott, C. Grebogi, and J. A. York, “Controlling chaos,” Phys. Rev. Lett.64, 1196-1199 (1990).

[24] S.Hayes, C. Grebogi, and E. Ott, "Communicating with chaos," Phys. Rev. Lett. 70,3031-3034 (1993).

[25] P. Colet, and R.Roy. "Digital communication with synchronized chaotic lasers," Opt. Lett. 19, 2056-2058 (1994).

[26] R. Daisy, and B. Fischer, "Synchronization of chaotic nonlinear optical oscillators," Opt. Comm. Vol 133, 282-286 (1997).

[27] M.R. Chatterjee and J.-J. Huang, "Demonstration of acousto-optic bistability and chaos by direct nonlinear circuit modeling," Appl. Opt. 31, 2506-2517 (1992).

[28] M. Chatterjee, and M. A. AI-Saedi, "Examination of chaotic signal encryption and recovery for secure communication using hybrid acousto-optic feedback, Opt.Eng” 50,55002-1 
$-055002-14,(2011)$.

[29] A.Korpel, “Acousto-optics," $2^{\text {nd }}$ edition (Marcel Dekker, New York,1997).

[30] P. Debye and F. W. Sears, "On the scattering of light bysupersonic waves," Proc. Nat.

Acad. Sci. 18, 409-414 (1932).

[31] R. Lucas and P. Biquard, "Optical properties of solid and liquid medias subjected to high-frequency elastic vibrations," Jour. De. Phys, et Rad., 3, 464-477 (1932).

[32] A. Korpel, "Selected appers on Acousto-Optics," SPIE milestone series, 16, 73-78 (1990).

[33] W.L. Bragg, "The Diffraction of Short Electromagnetic Waves by a Crystal," Proc.Camb. Phil. Soc. 17, 43-75 (1913).

[34] W.R. Klein and B.D. Cook, "Unified approach to ultrasonic light diffraction," IEEE Trans. Sonics Ultranson. SU-14, 123-133 (1967).

[35] S.-T. Chen, and M. R. Chatterjee "A numerical analysis and expository interpretation of the diffraction of light by ultrasonic waves in the Bragg and Raman-Nath regimes multiple scattering therory," IEEE Trans. On Education, 39, issue 1, 56-68 (1996).

[36] C.Webb, and J. Jones, "Handbook of Laser Technology and Applications - Vol. II," $1^{\text {st }}$ edition (IOP, Cornwall, UK, 2004).

[37] L. M. Pecora and T. L. Carroll, “"Synchronization in chaotic systems," Phys. Rev. Lett., 64, pp. 821-824, 1990.

[38] S. Donati and C. Mirasso, "Optical chaos and its applications to cryptography," IEEE J. 
Quant. Elec, 38, 1138-1196, (Sept. 2002).

[39] E. Mozdy, T.C. Newell, P.M. Alsing, V. Kovanis and A. Carrielides, "Synchronization and control in a unidirectional coupled array of chaotic diode resonators," Phy. Rev. E. (51), 5371-5376 (1995).

[40] K. M. Cuomo, A. V. Oppenheim and S. H. Strogratz, "Synchronization of Lorenz-based chaotic circuits with applications to communications," IEEE Trans. Circuits Syst. 40, 626-633 (1993).

[41] N. Grass, W. Kinzel, I. Kanter, M. Rosenbluh and L. Hykovich, "Synchronization of mutually versus unidirectionally coupled chaotic semiconductors," Opt.Comm. 267, 464-468 (2006).

[42] S. Banerjee, D. Ghosh, A. Ray and A.R. Chowdhury, "Synchronizationbetween two different time-delayed systems and image encryption,” Elp. 81, 562-568 (2008).

[43] M. R. Chatterjee and M. A. Al-Saedi, "Examination of the nonlinear dynamics of a chaotic acousto-optic Bragg modulator with feedback under signal encryption and decryption" Opt. Eng. 51(1) 018003 (2012). 